\title{
COMPUTATION OF $p$-UNITS IN RAY CLASS FIELDS OF REAL QUADRATIC NUMBER FIELDS
}

\author{
HUGO CHAPDELAINE
}

\begin{abstract}
Let $K$ be a real quadratic field, let $p$ be a prime number which is inert in $K$ and let $K_{p}$ be the completion of $K$ at $p$. As part of a Ph.D. thesis, we constructed a certain $p$-adic invariant $u \in K_{p}^{\times}$, and conjectured that $u$ is, in fact, a $p$-unit in a suitable narrow ray class field of $K$. In this paper we give numerical evidence in support of that conjecture. Our method of computation is similar to the one developed by Dasgupta and relies on partial modular symbols attached to Eisenstein series.
\end{abstract}

\section{INTRODUCTION}

Let $K$ be a real quadratic number field, let $p$ be a prime number which is inert in $K$ and let us denote by $K_{p}$ the completion of $K$ at $p$. In [DD06, Darmon and Dasgupta proposed a $p$-adic construction of elements $u_{\text {ring }} \in K_{p}^{\times}$and conjectured that $u_{\text {ring }}$ is a global $p$-unit lying in a suitable narrow ring class field of $K$. In Cha07a, we proposed a $p$-adic construction of elements $u_{\text {ray }} \in K_{p}^{\times}$and, in a similar way, we conjectured that $u_{\text {ray }}$ is, in fact, a global $p$-unit lying in a suitable narrow ray class field of $K$. Our $p$-adic invariant $u_{\text {ray }}$ can be viewed as a natural extension of the $p$-adic invariant $u_{\text {ring }}$. In Section 5.2 of [Cha07b, it is shown that the $p$-adic invariant $u_{\text {ray }}$ is compatible with the $p$-adic invariant $u_{\text {ring }}$.

We formulated a conjectural "Shimura reciprocity law" which describes the action of the Galois group $G_{K}=\operatorname{Gal}(\bar{K} / K)$ on $u_{\text {ray }}$ (see Conjecture 2.2). In Cha07b we showed that the truth of this conjecture implies the $p$-adic Gross-Stark conjecture for real quadratic number fields (see Theorem 5.1 of [Cha07b]). It is also expected that $u_{\text {ray }}$ can be written in terms of the Gross-Stark $p$-units $u_{G S}$ which were predicted by Conjecture 3.13 of Gro81. In Gro88, Gross proposed a $p$-adic formula for the image of $u_{G S}$ in $K_{p}^{\times} / \widehat{\mathcal{O}}_{K}^{\times}$where $\widehat{\mathcal{O}}_{K}^{\times}$is the closure of $\mathcal{O}_{K}^{\times}$in $K_{p}^{\times}$. Recently, Dasgupta in Das07b has proposed an exact $p$-adic formula for the image of $u_{G S}$ inside $K_{p}^{\times}$, which we denote by $u_{D}$. His formula allows him, at least conjecturally, to construct $p$-units in any $\mathrm{CM}$ abelian extension of a given totally real number field $L$. In the case where $L=K$, it can be shown that the $p$-adic invariant $u_{\text {ray }}$ can be written in terms of the $p$-adic invariants $u_{D}$. This fact will be addressed elsewhere.

Received by the editor November 14, 2007 and, in revised form, August 27, 2008.

2000 Mathematics Subject Classification. Primary 11S31.

Key words and phrases. $p$-adic Gross-Stark conjectures, explicit Class field theory, p-adic integration, Eisenstein series.

The author is grateful to the Max Planck Institut für Mathematik for the financial support during the writing of the paper.

(C)2009 American Mathematical Society Reverts to public domain 28 years from publication 
In Cha07b, we gave some theoretical evidence for the algebraicity of $u_{\text {ray }}$. Roughly speaking we showed that the norm of $u_{\text {ray }}$ down to a cyclotomic field is a product of normalized Gauss sums (see Theorem 6.1 of [Cha07b]). The norm here is taken analytically using the Shimura reciprocity law described above. Thus, this norm makes sense even though we do not know the algebraicity of $u_{\text {ray }}$.

In this paper we wish to give numerical evidence for the algebraicity of $u_{\text {ray }}$. We use an approach which is very similar to the one developed in Das07a. The computations were carried out on a computer at the Max Planck Institute for Mathematics. Our code was written in Magma. Some of the results of this paper can be found in a somewhat different form in the author's Ph.D. thesis Cha]. Finally, the author would like to acknowledge the efforts of the anonymous referee whose suggestions greatly improved the quality of the presentation.

\section{Definition of the Eisenstein SERIES And THE $p$-ADiC INVARIANT}

We now describe the construction of our $p$-adic invariant. Let $\left(p, N_{0}, f\right)$ be a triple of strictly positive integers which are pairwise coprime and where $p$ is a prime number. Let $K$ be a real quadratic number field with the ring of algebraic integers $\mathcal{O}_{K}$. We assume that the prime number $p$ is inert in $K$ and that there exists an ideal $\mathfrak{N}$ such that $\mathcal{O}_{K} / \mathfrak{N} \simeq \mathbb{Z} / N_{0} \mathbb{Z}$, the so-called "Heegner hypothesis".

Definition 2.1. We define $D\left(N_{0}, f\right)$ to be the free abelian group generated by the symbols of $\left\{\left[d_{0}, r\right]: 0<d_{0} \mid N_{0}, r \in \mathbb{Z} / f \mathbb{Z}\right\}$. A typical element $\delta \in D\left(N_{0}, f\right)$ will be denoted by

$$
\delta=\sum_{\substack{0<d_{0} \mid N_{0} \\ r \in \mathbb{Z} / f \mathbb{Z}}} n\left(d_{0}, r\right)\left[d_{0}, r\right],
$$

We have a natural action of $(\mathbb{Z} / f \mathbb{Z})^{\times}$on $D\left(N_{0}, f\right)$ given by $j \star\left[d_{0}, r\right]:=\left[d_{0}, j r\right]$ where $j \in(\mathbb{Z} / f \mathbb{Z})^{\times}$and we extend this action $\mathbb{Z}$-linearly to all of $D\left(N_{0}, f\right)$. We will use the shorthand notation

$$
\delta_{j}:=j \star \delta .
$$

Let $\delta=\sum_{d_{0} \mid N_{0}, r \in \mathbb{Z} / f \mathbb{Z}} n\left(d_{0}, r\right)\left[d_{0}, r\right] \in D\left(N_{0}, f\right)$ be such that the integers $n\left(d_{0}, r\right)$ are subject to the following three conditions:

(1) If $r \equiv 0(\bmod f)$, then for all $d_{0} \mid N_{0}$ we have $n\left(d_{0}, r\right)=0$.

(2) For all $r \in \mathbb{Z} / f \mathbb{Z}, \sum_{d_{0} \mid N_{0}} n\left(d_{0}, r\right) d_{0}=0$.

(3) For all $d_{0} \mid N_{0}$ and $r \in \mathbb{Z} / f \mathbb{Z}, n\left(d_{0}, p r\right)=n\left(d_{0}, r\right)$.

An element $\delta \in D\left(N_{0}, f\right)$ satisfying (1) and (2) and (3) will be called a good divisor for the triple $\left(N_{0}, f, p\right)$.

We want to associate Eisenstein series to any good divisor $\delta \in D\left(N_{0}, f\right)$. Let

$$
\begin{aligned}
E_{k}(r, \tau) & :=\left(\frac{(-1)^{k}(2 \pi i)^{k}}{(k-1) !}\right)^{-1} \sum_{\substack{(m, n) \in \mathbb{Z}^{2} \\
(0,0) \neq(m, n)}} \frac{e^{-2 \pi i m \frac{r}{f}}}{(m+n f \tau)^{k}} \\
& =\frac{-\widetilde{B}_{k}(-r / f)}{k}+\frac{1}{f^{k}} \sum_{b=0}^{f-1} e^{-2 \pi i b r / f} \sum_{m \geq 1} \sum_{n \geq 1} m^{k-1}\left(q_{n \tau+b / f}^{m}+(-1)^{k} q_{n \tau-b / f}^{m}\right),
\end{aligned}
$$


where $\tau \in \mathcal{H}=\{x+i y \in \mathbb{C}: y>0\}$ (the complex upper half-plane), $r \in \mathbb{Z} / f \mathbb{Z}$, $q_{n \tau+b / f}=e^{2 \pi i(n \tau+b / f)}$ and $\widetilde{B}_{k}(x):=B_{k}(\{x\})$, where $B_{k}(x)$ is the $k$-th Bernoulli polynomial and $0 \leq\{x\}<1$ is the fractional part of $x$. When $k \geq 3$ the convergence of the right-hand side of (2.1) is absolute and therefore $E_{k}(r, \tau)$ is a modular form of weight $k$ for the modular group $\Gamma_{1}(f)$. When $k=2$ the convergence is not absolute. Nevertheless, the corresponding $q$-expansion of (2.1) still converges and therefore we take it as the definition of $E_{2}(r, \tau)$. In the case where $r \not \equiv 0(\bmod f)$ and $k=2$, one can show that $E_{k}(r, \tau)$ satisfies the correct transformation formula and therefore corresponds to a holomorphic modular form of weight 2 for the modular group $\Gamma_{1}(f)$.

Next we want to associate Eisenstein series to a good divisor $\delta \in D\left(N_{0}, f\right)$.

Definition 2.2. Let $\delta=\sum_{d_{0} \mid N_{0}, r \in \mathbb{Z} / f \mathbb{Z}} n\left(d_{0}, r\right)\left[d_{0}, r\right] \in D\left(N_{0}, f\right)$ be a fixed good divisor. To any integer $k \geq 2$ we associate the Eisenstein series

$$
F_{k, \delta}(\tau):=\sum_{d_{0} \mid N_{0}, r \in \mathbb{Z} / f \mathbb{Z}} d_{0} n\left(d_{0}, r\right) E_{k}\left(r, d_{0} \tau\right),
$$

and its " $p$-stabilized" version,

$$
F_{k, \delta, p}(\tau):=F_{k, \delta}(\tau)-p^{k-1} F_{k, \delta}(p \tau) .
$$

For every $j \in(\mathbb{Z} / f \mathbb{Z})^{\times} /\langle p\rangle$ we also set

$$
\widetilde{F}_{k}(r, z):=-12 f F_{k, \delta_{r}}(z) \text { and } \widetilde{F}_{k, p}(r, z):=-12 f F_{k, \delta_{r}, p}(z) .
$$

Because the divisor $\delta$ satisfies the condition (2), the constant terms of the $q$ expansions of $\widetilde{F}_{k}(r, \tau)$ vanish at the cusps $\Gamma_{0}\left(f N_{0}\right)\{\infty\}$ where $\infty$ stands for the cusp $\frac{1}{0}$. It is "well known"that the period integrals

$$
\int_{c_{1}}^{c_{2}} z^{n} \widetilde{F}_{k}(r, z) d z
$$

are rational numbers for $c_{1}, c_{2} \in \Gamma_{0}\left(f N_{0}\right)\{\infty\}$ and $0 \leq n<k$. For explicit formulas of these periods given in terms of Dedekind sums see Proposition 11.1 of [Cha].

We need to introduce some background about $p$-adic integration. Let

$$
\mathbb{X}:=\left(\mathbb{Z}_{p} \times \mathbb{Z}_{p}\right) \backslash\left(p \mathbb{Z}_{p} \times p \mathbb{Z}_{p}\right) .
$$

Definition 2.3. Let $A$ be an abelian group. An $A$-valued distribution on $\mathbb{X}$ is a map

$$
\mu:\{\text { Compact open sets of } \mathbb{X}\} \rightarrow A
$$

which is finitely additive, i.e., for any disjoint union $\bigcup_{i=1}^{n} U_{i}$ of compact open sets of $\mathbb{X}$ we have

$$
\mu\left(\bigcup_{i=1}^{n} U_{i}\right)=\sum_{i=1}^{n} \mu\left(U_{i}\right) .
$$

A distribution is said to be a measure if $A$ can be chosen to be a bounded subgroup of $\mathbb{Q}_{p}$.

$$
\begin{aligned}
& \text { Let } \\
& \widetilde{\Gamma}_{0}:=\left\{\left(\begin{array}{ll}
a & b \\
c & d
\end{array}\right)=\gamma \in G L_{2}(\mathbb{Z}[1 / p]): \operatorname{det}(\gamma)>0, c \equiv 0 \quad\left(\bmod f N_{0}\right)\right\}
\end{aligned}
$$


and

$$
\Gamma_{1}:=\left\{\left(\begin{array}{cc}
a & b \\
c & d
\end{array}\right)=\gamma \in \widetilde{\Gamma}_{0}: \operatorname{det}(\gamma)=1, a \equiv 1 \quad\left(\bmod f N_{0}\right)\right\} .
$$

The next theorem is the crucial technical ingredient for the definition of our $p$-adic invariant.

Theorem 2.1. There exists a unique collection of p-adic measures $\widetilde{\mu}_{r}\left\{c_{1} \rightarrow c_{2}\right\}$ on $\left(\mathbb{Q}_{p} \times \mathbb{Q}_{p}\right) \backslash(0,0)$ taking values in $\mathbb{Z}$ and indexed by triples

$$
\left(r, c_{1}, c_{2}\right) \in(\mathbb{Z} / f \mathbb{Z})^{\times} /\langle\bar{p}\rangle \times \widetilde{\Gamma}_{0}\{\infty\} \times \widetilde{\Gamma}_{0}\{\infty\},
$$

such that:

(1) For every homogeneous polynomial $h(x, y) \in \mathbb{Z}_{p}[x, y]$ of degree $k-2$,

$$
\int_{\mathbb{X}} h(x, y) d \widetilde{\mu}_{r}\left\{c_{1} \rightarrow c_{2}\right\}(x, y)=\left(1-p^{k-2}\right) \int_{c_{1}}^{c_{2}} h(z, 1) \widetilde{F}_{k}(r, z) d z .
$$

(2) For all $\gamma \in \widetilde{\Gamma}_{0}$ and all compact open subsets $U \subseteq \mathbb{Q}_{p}^{2} \backslash(0,0)$,

$$
\widetilde{\mu}_{r}\left\{c_{1} \rightarrow c_{2}\right\}(U)=\widetilde{\mu}_{\gamma \star r}\left\{\gamma c_{1} \rightarrow \gamma c_{2}\right\}(\gamma U) .
$$

(3) For every homogeneous polynomial $h(x, y) \in \mathbb{Z}_{p}[x, y]$ of degree $k-2$,

$$
\int_{\mathbb{Z}_{p} \times \mathbb{Z}_{p}^{\times}} h(x, y) d \widetilde{\mu}_{r}\left\{c_{1} \rightarrow c_{2}\right\}(x, y)=\int_{c_{1}}^{c_{2}} h(z, 1) \widetilde{F}_{k, p}(r, z) d z .
$$

Proof. See Section 3 of Cha07a.

Now we introduce certain notions in order to give a precise definition of our $p$-adic invariant. Let $\mathcal{H}_{p}=\mathbb{P}^{1}\left(\mathbb{C}_{p}\right) \backslash \mathbb{P}^{1}\left(\mathbb{Q}_{p}\right)$ be the so-called $p$-adic upper halfplane endowed with its structure of rigid analytic space. Let $\mathcal{T}=\mathcal{T}_{0} \cup \mathcal{T}_{1}$ be the Bruhat-Tits tree for $P G L_{2}\left(\mathbb{Q}_{p}\right)$ where $\mathcal{T}_{0}$ corresponds to its set of vertices and $\mathcal{T}_{1}$ corresponds to its set of edges. We let $v_{0}$ be the standard vertex of $\mathcal{T}$ which corresponds to the homothety class of $\mathbb{Z}_{p} \oplus \mathbb{Z}_{p}$. Finally, we let red $: \mathcal{H}_{p} \rightarrow \mathcal{T}$ be the reduction map.

Definition 2.4. A point $\tau \in \mathcal{H}_{p}$ is said to be reduced if $\operatorname{red}(\tau)=v_{0}$. This is equivalent to saying that $|\tau-t|_{p} \geq 1$ for $t=0,1, \ldots, p-1$ and $|\tau|_{p} \leq 1$ where ||$_{p}$ stands for the $p$-adic valuation on $\mathbb{C}_{p}$ normalized in a such a way that $|p|_{p}=\frac{1}{p}$.

For a short introduction to the objects defined in the previous paragraph see Chapter 5 of [Dar04].

Let $K_{p}$ be the completion of $K$ at the prime $p$. Note that $\mathcal{H}_{p} \cap K \neq \emptyset$. For certain pairs $(r, \tau) \in \mathbb{Z} / f \mathbb{Z} \times\left(\mathcal{H}_{p} \cap K\right)$ we want to associate a $p$-adic invariant $u(r, \tau) \in K_{p}^{\times}$. Let us fix an embedding $K \subseteq \mathbb{R}$. For every $\tau \in K-\mathbb{Q}$ we define the order $\mathcal{O}_{\tau}$ as $\operatorname{End}_{K}\left(\Lambda_{\tau}\right)$ where $\Lambda_{\tau}$ is the lattice $\mathbb{Z}+\tau \mathbb{Z}$. For an element $\tau \in K \backslash \mathbb{Q}$ we define $Q_{\tau}(x, y):=A x^{2}+B x y+C y^{2}$ to be the unique normalized binary quadratic form such that $A \tau^{2}+B \tau+C=0, \operatorname{gcd}(A, B, C)=1$ and $A>0$. We define the set

$$
\mathcal{H}_{p}\left(N_{0}, f\right):=\left\{\tau \in \mathcal{H}_{p} \cap K: \mathcal{O}_{\tau}^{(p)}=\mathcal{O}_{N_{0} \tau}^{(p)}=\mathcal{O}_{K}^{(p)},\left(\Lambda_{\tau}^{(p)}, f \mathcal{O}_{K}^{(p)}\right)=1, \tau-\tau^{\sigma}>0\right\},
$$

where for a $\mathbb{Z}$-module $M, M^{(p)}:=M \otimes_{\mathbb{Z}} \mathbb{Z}\left[\frac{1}{p}\right]$. The notation $\left(\Lambda_{\tau}^{(p)}, f \mathcal{O}_{K}^{(p)}\right)=1$ means that $\Lambda_{\tau}^{(p)}=\frac{\mathfrak{a}}{\mathfrak{b}}$ where $\mathfrak{a}, \mathfrak{b}$ are integral $\mathcal{O}_{K}^{(p)}$-ideals which are coprime to $f$. This is equivalent to saying that $(A, f)=1$ where $A$ is the leading coefficient of $Q_{\tau}(x, y)=A x^{2}+B x y+C y^{2}$. 
We are now ready to define our $p$-adic invariant.

Definition 2.5. Let $(r, \tau) \in(\mathbb{Z} / f \mathbb{Z})^{\times} \times \mathcal{H}_{p}\left(N_{0}, f\right)$ where $\tau$ is reduced. We define the $p$-adic invariant

$$
u\left(\delta_{r}, \tau\right)=u(r, \tau):=p^{\psi_{r}\left\{\infty \rightarrow \gamma_{\tau} \infty\right\}} \mathcal{J}_{\mathbb{X}}(x-\tau y) d \widetilde{\mu}_{r}\left\{\infty \rightarrow \gamma_{\tau} \infty\right\}(x, y) \in K_{p}^{\times},
$$

where $\gamma_{\tau}$ is an oriented generator of the stabilizer of $\tau$ under the action of $\Gamma_{1}$, i.e., $\gamma_{\tau}$ is chosen in such a way that it generates the quotient $\operatorname{Stab}_{\Gamma_{1}}(\tau) /\langle \pm 1\rangle \simeq \mathbb{Z}$ and

$$
\gamma_{\tau}\left(\begin{array}{c}
\tau \\
1
\end{array}\right)=\epsilon\left(\begin{array}{c}
\tau \\
1
\end{array}\right)
$$

with $\epsilon>1$. For any pair of cusps $c_{1}, c_{2} \in \Gamma_{0}\left(f N_{0}\right)\{\infty\}$, the quantity $\psi_{r}\left\{c_{1} \rightarrow c_{2}\right\}$ is defined by the integral

$$
\psi_{r}\left\{c_{1} \rightarrow c_{2}\right\}:=\frac{1}{2 \pi i} \int_{c_{1}}^{c_{2}} \widetilde{F}_{2}(r, \tau) d \tau
$$

where the complex line integral on the right-hand side is taken along the unique geodesic $C$ in the complex upper half-plane $\mathcal{H}$ connecting the cusps $c_{1}$ and $c_{2}$.

It is explained in Section 2 of Cha07a that the rational number $\psi_{r}\left\{c_{1} \rightarrow c_{2}\right\}$ is in fact always an integer. Some explanations about the multiplicative integral appearing in (2.3) are in order. This $p$-adic integral is defined by

$$
f_{\mathbb{X}}(x-\tau y) d \widetilde{\mu}_{r}\left\{\infty \rightarrow \gamma_{\tau} \infty\right\}(x, y):=\lim _{\|\mathcal{U}\| \rightarrow 0} \prod_{U \in \mathcal{U}}\left(x_{U}-\tau y_{U}\right)^{\widetilde{\mu}_{r}\left\{\infty \rightarrow \gamma_{\tau} \infty\right\}(U)} \in K_{p}^{\times},
$$

where $\mathcal{U}$ is a cover of $\mathbb{X}$ by disjoint compact open sets, $\left(x_{U}, y_{U}\right)$ is an arbitrary point of $U \in \mathcal{U}$, and the $p$-adic limit is taken over increasingly fine covers $\mathcal{U}$. The product in (2.5) makes sense since the measures $\widetilde{\mu}_{r}\left\{c_{1} \rightarrow c_{2}\right\}$ are $\mathbb{Z}$-valued and not only $\mathbb{Z}_{p}$-valued.

2.1. Statement of the conjectures. We conjecture that the element $u(r, \tau)$ lies in the narrow ray class field $K$ of conductor $f$ which we denote by $K(f \infty)$. More precisely:

Conjecture 2.1. Let $L:=K(f \infty)^{\text {Frob }(\mathfrak{p} / \wp)}$ where $\wp=p \mathcal{O}_{K}$ and $\mathfrak{p}$ is a prime ideal of $K(f \infty)$ above $\wp$. Then the element $u(r, \tau) \in K_{p}^{\times}$is a "strong $p$-unit" in L, i.e., an element of $\mathcal{O}_{L}\left[\frac{1}{p}\right]^{\times}$, such that $|u(r, \tau)|_{\nu}=1$ for all infinite places $\nu$ of $L$.

The appellation $p$-adic invariant for the quantity $u(r, \tau)$ is appropriate in light of the following theorem.

Theorem 2.2. Let $(r, \tau),\left(r^{\prime}, \tau^{\prime}\right) \in(\mathbb{Z} / f \mathbb{Z})^{\times} \times \mathcal{H}_{p}\left(N_{0}, f\right)$ where $\tau$ and $\tau^{\prime}$ are reduced. Furthermore, assume that

$$
\gamma \star(r, \tau):=(\gamma \star r, \gamma \tau)=\left(r^{\prime}, \tau^{\prime}\right), \quad \gamma=\left(\begin{array}{ll}
a & b \\
c & d
\end{array}\right) \in \widetilde{\Gamma}_{0} .
$$

Then

$$
u(r, \tau) \equiv u\left(r^{\prime}, \tau^{\prime}\right) \quad\left(\bmod \left(K_{p}^{\times}\right)_{t o r}\right),
$$

where $\left(K_{p}^{\times}\right)_{\text {tor }}=\mu_{p^{2}-1}$.

Proof. See Theorem 1.2 of [Cha07a]. 
It is a natural question to ask if (2.6) remains valid without working modulo $\mu_{p^{2}-1}$. The author did not attempt to prove it but numerical examples suggest that this refinement is true.

Now we would like to state a conjectural "Shimura reciprocity law"which describes the action of $\operatorname{Gal}(L / K)$ on the $p$-adic invariant $u(r, \tau)$. We need to set up some definitions. Define the set $\mathcal{M}_{K}\left(N_{0}, f, p\right)$ to be

$$
\begin{aligned}
& \left\{(L, M) \text { : pairs of } \mathbb{Z}\left[\frac{1}{p}\right] \text {-modules of rank } 2 \text { in } K, \operatorname{End}_{K}(L)=\operatorname{End}_{K}(M)=\mathcal{O}_{K}^{(p)},\right. \\
& \left.\qquad\left(L, f \mathcal{O}_{K}^{(p)}\right)=\left(M, f \mathcal{O}_{K}^{(p)}\right)=1 \text { and } L / M \simeq \mathbb{Z} / N_{0} \mathbb{Z}\right\} .
\end{aligned}
$$

We have a natural equivalence relation on $\mathcal{M}\left(N_{0}, f, p\right)$ which we denote by $\sim$, where $(L, M) \sim\left(L^{\prime}, M^{\prime}\right)$ if and only if there exists a totally positive element $\lambda \in 1+f L^{\prime-1}$ such that $(L, M)=\left(\lambda L^{\prime}, \lambda M^{\prime}\right)$.

Proposition 2.1. There exists a natural bijection of sets, which we denote by $\psi$, between

$$
\psi:\left((\mathbb{Z} / f \mathbb{Z})^{\times} \times \mathcal{H}_{p}\left(N_{0}, f\right)\right) / \widetilde{\Gamma}_{0} \longrightarrow \mathcal{M}_{K}\left(N_{0}, f, p\right) / \sim .
$$

Proof. See Proposition 4.1 of Cha07a].

Using Proposition 2.1 one may define an action of $G_{L / K}$ on

$$
\left((\mathbb{Z} / f \mathbb{Z})^{\times} \times \mathcal{H}_{p}\left(N_{0}, f\right)\right) / \widetilde{\Gamma}_{0} .
$$

In order to do so, we first need to recall a reciprocity isomorphism given by Class field theory in our context.

Let $\mathfrak{f}=f \mathcal{O}_{K}$. We let $I_{\mathcal{O}_{K}}(\mathfrak{f})$ be the monoid of integral ideals coprime to $\mathfrak{f}$. Let $\mathfrak{a}, \mathfrak{b} \in I_{\mathcal{O}_{K}}(\mathfrak{f})$. We say that $\mathfrak{a} \sim_{\mathfrak{f}} \mathfrak{b}$ if and only if there exists an element $\lambda \in 1+\mathfrak{f a}^{-1}$, $\lambda \gg 0$ (totally positive), such that $\lambda \mathfrak{a}=\mathfrak{b}$. Also, let

$$
P_{\mathcal{O}_{K}}(\mathfrak{f} \infty)=\left\{\frac{\alpha}{\beta} \in K: \alpha, \beta \in \mathcal{O}_{K}, \alpha \equiv \beta(\bmod \mathfrak{f}), \frac{\alpha}{\beta} \gg 0\right\} .
$$

It is easy to see that for $\mathfrak{a}, \mathfrak{b} \in I_{\mathcal{O}_{K}}(\mathfrak{f}), \mathfrak{a} \sim_{\mathfrak{f}} \mathfrak{b}$ if and only if there exists a $\lambda \in$ $P_{\mathcal{O}_{K}}(\mathfrak{f} \infty)$ such that $\lambda \mathfrak{a}=\mathfrak{b}$. We can thus think of $I_{\mathcal{O}_{K}}(\mathfrak{f}) / \sim_{\mathfrak{f}}$ as $I_{\mathcal{O}_{K}}(\mathfrak{f}) / P_{\mathcal{O}_{K}}(\mathfrak{f} \infty)$. Class field theory gives us a reciprocity isomorphism

$$
\text { rec }: G_{L / K} \rightarrow I_{\mathcal{O}_{K}}(\mathfrak{f}) /\left\langle P_{\mathcal{O}_{K}}(\mathfrak{f} \infty), p \mathcal{O}_{K}\right\rangle \text {. }
$$

We define an action of $G_{L / K}$ on $\left((\mathbb{Z} / f \mathbb{Z})^{\times} \times \mathcal{H}_{p}\left(N_{0}, f\right)\right) / \widetilde{\Gamma}_{0}$ given by the following rule: Let $[(r, \tau)] \in\left((\mathbb{Z} / f \mathbb{Z})^{\times} \times \mathcal{H}_{p}\left(N_{0}, f\right)\right) / \widetilde{\Gamma}_{0}$ and $\psi[(r, \tau)]=[(L, M)]$. Now define

$$
\operatorname{rec}^{-1}(\mathfrak{b}) \star[(r, \tau)]:=\psi^{-1}[(\mathfrak{b} L, \mathfrak{b} M)]
$$

We can now state a refinement of Conjecture 2.1

Conjecture 2.2. Let $(r, \tau) \in(\mathbb{Z} / f \mathbb{Z})^{\times} \times \mathcal{H}_{p}\left(N_{0}, f\right)$ where $\operatorname{red}(\tau)=v_{0}$. Then

$$
u(r, \tau) \in \mathcal{O}_{L}\left[\frac{1}{p}\right]^{\times},
$$

where $L=K(\mathfrak{f} \infty)^{\left\langle F r_{\wp}\right\rangle}, \mathfrak{f}=f \mathcal{O}_{K}$ and $\wp=p \mathcal{O}_{K}$. Moreover, we have a Shimura reciprocity law: for $\sigma \in G_{L / K}$,

$$
u(k, \tau)^{\sigma^{-1}}=u\left(k^{\prime}, \tau^{\prime}\right) \quad\left(\bmod \mu_{p^{2}-1}\right),
$$

where $\sigma \star[(k, \tau)]=\left[\left(k^{\prime}, \tau^{\prime}\right)\right]$. 
Remark 2.1. Note that the Shimura reciprocity law is only stated modulo (mod $\left.\mu_{p^{2}-1}\right)$ since the pair $\left(r^{\prime}, \tau^{\prime}\right)$ (for $\tau^{\prime}$ reduced) is only well defined modulo the left action of $\widetilde{\Gamma}_{0} \cap S L_{2}(\mathbb{Z})$.

The goal of this article is the numerical verification of Conjecture 2.2 in many cases.

\section{Dedekind sums And Distributions}

In this section, we first recall certain facts about Bernoulli polynomials. We then introduce certain Dedekind sums and distributions that play a key role for the explicit computation of $u(r, \tau)$.

Definition 3.1. We define the $n$-th Bernoulli polynomial $B_{n}(x)$ (for $n \geq 1$ ) by the identity

$$
\frac{t e^{x t}}{e^{t}-1}=\sum_{n \geq 0} B_{n}(x) \frac{t^{n}}{n !}
$$

The $n$-th Bernoulli number $B_{n}$ is defined as $B_{n}:=B_{n}(0)$. A direct computation using (3.1) shows that

$$
B_{n}(x)=\sum_{i=0}^{n}\left(\begin{array}{c}
n \\
i
\end{array}\right) B_{i} x^{n-i} .
$$

For $n \geq 2$, we also define the $n$-th "periodic Bernoulli polynomial" as

$$
\widetilde{B}_{n}(x):=B_{n}(\{x\})
$$

where $\{x\}=x-[x]$ is the fractional part of $x$. For $n=1$, we define

$$
\widetilde{B}_{1}(x):=\{x\}-\frac{1}{2}+\frac{\mathbb{1}_{\mathbb{Z}}(x)}{2},
$$

where $\mathbb{1}_{\mathbb{Z}}(x)$ is the characteristic function of the set $\mathbb{Z}, \widetilde{B}_{1}(x)$ being the famous sawtooth function.

Using (3.1), one can deduce the useful formula $B_{n}(1-x)=(-1)^{n} B_{n}(x)$. From this, one sees that $\widetilde{B}_{n}(-x)=(-1)^{n} \widetilde{B}_{n}(x)$.

We can now introduce the Dedekind sums that will play a crucial role for the computation of our $p$-adic invariant.

Definition 3.2. Let $a$ and $0<c$ be two integers which are not necessarily coprime and assume that $f \mid c$. Let $s, t \geq 1$ be integers and choose a residue class $r \in \mathbb{Z} / f \mathbb{Z}$. We define the Dedekind sum

$$
D_{s, t}^{r}(\bmod f)(a, c):=c^{s-1} \sum_{\substack{1 \leq h \leq c \\ h \equiv r}} \frac{\widetilde{B}_{s}(h / c)}{s} \frac{\widetilde{B}_{t}(h a / c)}{t},
$$

where $\widetilde{B}_{n}$ is the $n$-th periodic Bernoulli polynomial.

Let $e \geq 1$ be a positive integer divisible by $f N_{0}$ but not by $p$ and let

$$
Z=\underbrace{\lim }_{n} \mathbb{Z} / e p^{n} \mathbb{Z} \simeq \mathbb{Z} / e \mathbb{Z} \times \mathbb{Z}_{p}
$$


Definition 3.3. Let $\delta=\sum_{d_{0} \mid N_{0}, r \in(\mathbb{Z} / f \mathbb{Z}) \times} n\left(d_{0}, r\right)\left[d_{0}, r\right] \in D\left(N_{0}, f\right)$ be a good divisor with respect to the data $\left(N_{0}, f, p\right)$. For each integer $k \geq 1$ and $r \in \mathbb{Z} / f \mathbb{Z}$ we define a distribution $\mathcal{F}_{k, r}$ on $Z$ by the rule

$$
\mathcal{F}_{k, r}\left(a+e p^{n} Z\right):=\sum_{d_{0} \mid N_{0}} n\left(d_{0}, r\right)\left(\frac{e p^{n}}{d_{0}}\right)^{k-1} \frac{\widetilde{B}_{k}\left(\frac{a}{e p^{n} / d_{0}}\right)}{k},
$$

where $a$ is any integer.

We have a natural action of $(\mathbb{Z} / f \mathbb{Z})^{\times}$on the distributions $\mathcal{F}_{k, r}$ given by $j \star \mathcal{F}_{k, r}=$ $\mathcal{F}_{k, r j}$. Note that for any compact open set $U \subseteq Z$ we have

$$
\mathcal{F}_{k, r}(p U)=p^{k-1} \mathcal{F}_{k, r}(U) .
$$

For $x \in Z$ we let $x_{p}$ denote the projection of $x$ on $\mathbb{Z}_{p}$.

Proposition 3.1. The distributions $\mathcal{F}_{k, r}$ are $\mathbb{Z}_{p}$-valued measures, and for every compact open set $U \subseteq Z$, every integer $k \geq 1$ and residue class $r \in \mathbb{Z} / f \mathbb{Z}$, we have

$$
\mathcal{F}_{k, r}(U)=\int_{U} x_{p}^{k-1} d \mathcal{F}_{1, r}(x) .
$$

Proof. See Proposition 3.1 of Das07a.

\section{EXPLICIT FORMULAS}

In this section we record various explicit formulas which are used for the computation of $u(r, \tau)$.

Let us start by giving an explicit formula for the measures $\widetilde{\mu}_{j}\left\{\infty \rightarrow \frac{a}{c}\right\}$ when evaluated on the compact open sets of the form $\left(u+p^{s} \mathbb{Z}_{p}\right) \times\left(v+p^{s} \mathbb{Z}_{p}\right)$ for $u, v \in \mathbb{Z}$ and $(u, v, p)=1$.

Proposition 4.1. Let $u, v \in \mathbb{Z}$ such that $(u, v) \in \mathbb{X}$. For a positive integer $s$, let $U_{u, v, s}$ denote the ball of radius $\frac{1}{p^{s}}$ around $(u, v) \in \mathbb{X}$, i.e.,

$$
U_{u, v, s}=\left(u+p^{s} \mathbb{Z}_{p}\right) \times\left(v+p^{s} \mathbb{Z}_{p}\right) \subseteq \mathbb{X} .
$$

Let $\frac{a}{c} \in \Gamma_{0}\left(f N_{0}\right)\{\infty\}$. Then

$$
\begin{aligned}
& \widetilde{\mu}_{j}\left\{\infty \rightarrow \frac{a}{c}\right\}\left(U_{u, v, s}\right) \\
& =-12 \sum_{d_{0}, r} n\left(d_{0}, r\right) \sum_{\substack{1 \leq h \leq p^{s} c / d_{0} \\
h \equiv f v \\
h \equiv r j}} \widetilde{B}_{1}\left(\frac{a h}{\left.\bmod ^{s} f\right)}\right)
\end{aligned}
$$

Proof. See the proof of Propostion 3.2 in Das07b] or that of Proposition 14.1 in Cha.

We now record explicit formulas for the moments of the measures $\widetilde{\mu}_{j}\left\{\infty \rightarrow \frac{a}{c}\right\}$ appearing in Theorem 2.1. These formulas are crucial for the explicit computation of the $p$-adic invariant $u(r, \tau)$ appearing in (2.3). 
Proposition 4.2. Let $\xi=\frac{a}{c} \in \Gamma_{0}\left(f N_{0}\right)\{\infty\}$ with $c \geq 1$ and let $\widetilde{\mu}_{j}\left\{i \infty \rightarrow \frac{a}{c}\right\}$ be as in Theorem 2.1, Then we have

$$
\begin{aligned}
\int_{\mathbb{X}} x^{n} y^{m} d \widetilde{\mu}_{j}\left\{\infty \rightarrow \frac{a}{c}\right\}(x, y)=\left(1-p^{n+m}\right) \int_{\xi}^{\infty} z^{n} \widetilde{F}_{n+m+2}(j, z) d z \\
=\frac{-12}{f^{n+m}}\left(1-p^{n+m}\right) \sum_{l=0}^{n}\left(\begin{array}{c}
n \\
l
\end{array}\right)\left(\frac{a}{c}\right)^{n-l}(-1)^{l} \\
\cdot \sum_{d_{0} \mid N_{0}, r \in \mathbb{Z} / f \mathbb{Z}} n\left(d_{0}, r\right) d_{0}^{-l} D_{n+m-l+1, l+1}^{j r}\left(a, c / d_{0}\right) .
\end{aligned}
$$

Proof. See Proposition 11.5 in Cha].

Proposition 4.3. Let $\gamma \in \Gamma_{0}\left(f N_{0}\right)$ where $\gamma=\left(\begin{array}{ll}a & b \\ c & d\end{array}\right)$ and $c \geq 1$. Then we have

$$
\psi_{j}\{\infty \rightarrow \gamma \infty\}=\int_{\infty}^{\gamma \infty} \widetilde{F}_{2}(j, \tau) d \tau=-12 \sum_{d_{0} \mid N_{0}, r \in \mathbb{Z} / f \mathbb{Z}} n\left(d_{0}, r\right) D_{1,1}^{r j} \quad(\bmod f)\left(a, c / d_{0}\right) .
$$

Proof. Use the fact that $\operatorname{d} \log \beta_{\delta_{j}}(\tau)=2 \pi i \widetilde{F}_{2}(j, \tau)$ in Proposition 5.4 of [Cha].

Proposition 4.4. Let $\widetilde{\mu}_{j}\left\{\infty \rightarrow \frac{a}{c}\right\}$ be as in Theorem 2.1 with $c \geq 1$. Then we have

$$
\begin{aligned}
\int_{\mathbb{Z}_{p} \times \mathbb{Z}_{p}^{\times}} & x^{n} y^{m} d \widetilde{\mu}_{j}\left\{\infty \rightarrow \frac{a}{c}\right\}(x, y)=\int_{\xi}^{\infty} z^{n} \widetilde{F}_{n+m+2, p}(j, z) d z \\
= & \frac{-12}{f^{n+m}} \sum_{l=0}^{n}\left(\begin{array}{l}
n \\
l
\end{array}\right)\left(\frac{a}{c}\right)^{n-l}(-1)^{l} \\
& \cdot \sum_{d_{0} \mid N_{0}, r \in \mathbb{Z} / f \mathbb{Z}} n\left(d_{0}, r\right) d_{0}^{-l}\left(D_{n+m-l+1, l+1}^{j r}\left(a, c / d_{0}\right)\right. \\
& \quad-p^{n+m-l} D_{n+m-l+1, l+1}^{j r}(\bmod f) \\
&
\end{aligned}
$$

Proof. See Proposition 11.6 in Cha].

\section{Method to compute $u(r, \tau)$}

Conjecture 2.1 asserts the existence of strong $p$-units in abelian extensions of real quadratic number fields. In order to make sure that such units exist, one needs to impose a number of conditions on the real quadratic field $K$. To fix the ideas, let us assume that $f=3, N_{0}=4$ and that

$$
\delta=2[1,1]-3[2,1]+1[4,1] \in D(4,3) .
$$

Let $K=\mathbb{Q}(\sqrt{D})$ be a real quadratic field where $D=\operatorname{disc}(K)$. In order to facilitate the existence of nontrivial strong $p$-units in $K(3 \infty)$ one requires the following:

(1) $(D, 3)=1(3$ is unramified in $K)$.

(2) $D \equiv 1(\bmod 8)(2$ is split in $K)$.

(3) $\left(\frac{D}{p}\right)=-1(p$ is inert in $\mathbb{Q}(\sqrt{D}))$.

(4) The index $n=\left[\mathcal{O}_{K}\left[\frac{1}{p}\right](3)^{\times}: \mathcal{O}_{K}\left[\frac{1}{p}\right](3 \infty)^{\times}\right]$is equal to 1 or 2 . The group $\mathcal{O}_{K}\left[\frac{1}{p}\right](3)^{\times}$(resp. $\left.\mathcal{O}_{K}\left[\frac{1}{p}\right](3 \infty)^{\times}\right)$stands for the group of units (resp. totally positive units) of $\mathcal{O}_{K}\left[\frac{1}{p}\right]$ which are congruent to 1 modulo 3 . 
Remark 5.1. For a general integer $f>0$, if the index $n=\left[\mathcal{O}_{K}\left[\frac{1}{p}\right](f)^{\times}: \mathcal{O}_{K}\left[\frac{1}{p}\right](f \infty)^{\times}\right]$ is equal to 4 , one can prove that $K(f \infty)^{\operatorname{Frob}(\mathfrak{p} / \wp)}=K(f)^{\operatorname{Frob}(\mathfrak{p} / \wp)}$ is a totally real field $\left(\wp=p \mathcal{O}_{K}\right.$ and $\mathfrak{p}$ is a prime ideal of $K(f \infty)$ above $\left.\wp\right)$. When the class field $K(f \infty)^{\operatorname{Frob}(\mathfrak{p} / \wp)}$ is totally real, it is easy to see there are no strong $p$-units in $K(f \infty)^{F r o b(\mathfrak{p} / \wp)}$ other than $\{ \pm 1\}$. See Section 7 for more details.

A discriminant $D>0$ satisfying these four conditions will be called admissible. A congruence modulo 3 shows that there exists no unit $\epsilon \in \mathcal{O}_{K}(3)^{\times}$such that $\mathbf{N}(\epsilon)=-1$. Therefore, the fourth condition is always satisfied and can thus be dropped. Using class field theory, one deduces that

$$
K(3 \infty) \supseteq K\left(\zeta_{3}\right)=K(\sqrt{-3}),
$$

where $\zeta_{3}=e^{2 \pi i / 3}$. From (5.1), it follows that $K(3 \infty)=K(\sqrt{-3})$ when the narrow ray class group $K$ of conductor 3 has order 2 .

Conjecture 2.1predicts that the strong $p$-units arising from our construction lie in $K(3 \infty)^{\langle F r o b(\mathfrak{p} / \wp)\rangle}$. Since we would like our strong $p$-units to be primitive elements of $K(3 \infty)$ over $K$, we will impose the additional condition that $\operatorname{Frob}(\mathfrak{p} / \wp)=1$. This is equivalent by class field theory to the congruence $p \equiv 1(\bmod 3)$. Note that the last condition is a necessary condition for the strong $p$-units to be primitive, but it is in general not sufficient as the tables show. The author does not know of a condition which is sufficient.

Let us fix an embedding $K \subseteq \mathbb{R}$. We define $I_{K}(3)$ to be the group of fractional ideals of $K$ coprime to 3 and we let $P_{K, 1}(3 \infty)$ be the group of principal fractional ideals of $K$ which can be generated by a totally positive element congruent to 1 modulo 3. We also define $P_{K, 1}(3)$ to be the group of principal fractional ideals of $K$ which can be generated by an element congruent to 1 modulo 3 . Let $n=\left[\mathcal{O}_{K}(3)^{\times}\right.$: $\mathcal{O}_{K}(3 \infty)^{\times}$]. Because $f=3$, we always have that $n=1$ or 2 . A calculation shows that the quotient $P_{K, 1}(3) / P_{K, 1}(3 \infty) \simeq(\mathbb{Z} / 2 \mathbb{Z})^{3-n}$. When $n=1$ the quotient group $P_{K, 1}(3) / P_{K, 1}(3 \infty) \simeq(\mathbb{Z} / 2 \mathbb{Z})^{2}$ can be generated by the ideal classes $(1+3 \sqrt{D}) \mathcal{O}_{K}$ and $(1-3 \sqrt{D}) \mathcal{O}_{K}$. When $n=2$ there exists a unit $\epsilon \in \mathcal{O}_{K}(3)^{\times}$such that $\epsilon<0$ and $\epsilon^{\sigma}>0$ and therefore the ideal $(1-3 \sqrt{D}) \mathcal{O}_{K}=\epsilon(1-3 \sqrt{D}) \mathcal{O}_{K}$ is equivalent to $(1+3 \sqrt{D}) \mathcal{O}_{K}$ modulo $P_{K, 1}(3 \infty)$.

For every admissible discriminant $D$ the narrow class group of $K=\mathbb{Q}(\sqrt{D})$ of conductor 3 is given by $I_{K}(3) / P_{K, 1}(3 \infty)$. Let $J:=\langle 2, \omega\rangle$ be a prime ideal of $K$ above 2 , where $\omega=\frac{1+\sqrt{D}}{2}$. For every ideal class $C \in I_{K}(3) / P_{K, 1}(3 \infty)$ we pick an ideal $\mathfrak{a}_{C} \in C$. Since the quotient $\mathfrak{a}_{C} /\left(\mathfrak{a}_{C} J^{2}\right)$ is isomorphic to $\mathbb{Z} / 4 \mathbb{Z}$ we can always find elements $\omega_{1}, \omega_{2} \in \mathcal{O}_{K}$, such that

$$
\mathfrak{a}_{C}=\mathbb{Z} \omega_{1}+\mathbb{Z} \omega_{2}, \quad \mathfrak{a}_{C} J^{2}=\mathbb{Z} \omega_{1}+\mathbb{Z} 4 \omega_{2} \quad \text { and } \quad \omega_{1}>0 .
$$

Moreover, an easy calculation shows that one can always choose $\omega_{1}$ in such a way that

$$
\omega_{1} \equiv \text { integer } \quad(\bmod 3)
$$

Now assume that $\omega_{1}, \omega_{2}$ satisfy (5.2) and (5.3). Then if we set $\tau=\frac{\omega_{2}}{\omega_{1}}$ we readily see that $r \Lambda_{\tau}$ is equivalent to $\mathfrak{a}_{C}$ modulo $P_{K, 1}(3)$, where $\Lambda_{\tau}=\mathbb{Z}+\tau \mathbb{Z}$. Note that $r \Lambda_{\tau}$ is equivalent to $\mathfrak{a}_{C}$ modulo $P_{K, 1}(3 \infty)$ precisely when $\mathbf{N}_{K / \mathbb{Q}}\left(\omega_{1}\right)>0$. We set

$$
\mathfrak{s}=\operatorname{sign}\left(\mathbf{N}_{K / \mathbb{Q}}\left(\omega_{1}\right)\right) \in\{ \pm 1\} .
$$


We require two more conditions on the choice of $\tau$, namely that $|\tau-i|_{p}=1$ for $0 \leq i \leq p-1$ (i.e., $\tau$ is reduced) and also that $\tau-\tau^{\sigma}>0(\tau$ is oriented $)$ where $\sigma$ is the nontrivial automorphism of $K$. Let $\epsilon>1$ be such that $\mathcal{O}_{K}(3 \infty)^{\times}=\epsilon^{\mathbb{Z}}$. Let $\gamma_{\tau}$ be the matrix corresponding to the action of $\epsilon$ on $\Lambda_{\tau}$ with respect to the ordered basis $\{\tau, 1\}$ :

$$
\gamma_{\tau}\left(\begin{array}{c}
\tau \\
1
\end{array}\right)=\epsilon\left(\begin{array}{c}
\tau \\
1
\end{array}\right)
$$

If we write $\gamma_{\tau}=\left(\begin{array}{ll}a & b \\ c & d\end{array}\right)$, then one can verify that $c \equiv 0(\bmod 12)$ and $d \equiv 1$ $(\bmod 3)$. For this ideal class $C$ we associate the following $p$-adic invariant

$$
u(C):=u(r, \tau)^{\mathfrak{s}}=\left(p^{\psi_{r}\left\{\infty \rightarrow \gamma_{\tau} \infty\right\}} \oint_{\mathbb{X}}(x-\tau y) d \widetilde{\mu}_{r}\left\{\infty \rightarrow \gamma_{\tau} \infty\right\}(x, y)\right)^{\mathfrak{s}} \in K_{p}^{\times} .
$$

Note that the pair $(r, \tau)$ (for $\tau$ reduced) which is associated to the ideal class $C$ is only well defined modulo the action of $\widetilde{\Gamma}_{0} \cap S L_{2}(\mathbb{Z})$ and therefore, in light of Theorem 2.2 the invariant $u(C)$ is only well defined modulo $\mu_{p^{2}-1}$ even though the quantity $u(r, \tau)$ is a well defined element of $K_{p}^{\times}$.

Assuming Conjecture 2.1 we may deduce that the polynomial

$$
P_{D}(x):=\prod_{C \in I_{K}(3) / P_{K, 1}(3 \infty)}(x-u(C)) \in K_{p}[x]
$$

has coefficients in $\mathcal{O}_{K}\left[\frac{1}{p}\right]$. Let $c_{\infty}$ be a complex conjugation of $K(f \infty) / K$ (a complex conjugation of $K(f \infty)$ is not necessarily unique); then Conjecture 2.1 predicts that $u(r, \tau)^{c_{\infty}}=u(r, \tau)^{-1}$. Because of this, the polynomial $P_{D}(x)$ is a palindromic polynomial. Because of the presence of the factor 12 in the definition of $\widetilde{F}_{k}(j, \tau)$, it turns out that very often our units $u(C)$ are powers of other units. For this reason, for every admissible $D$, we define a certain integer $n_{D}$. The integer $n_{D}$ is chosen to be the largest positive integer dividing 12 for which

$$
\begin{aligned}
2 \widetilde{P}_{D}(x) \in\left\{f(x) \in \mathbb{Z}\left[\frac{1}{p}\right][\sqrt{D}][x]: f(x)=\sum_{i} \frac{a_{i}+b_{i} \sqrt{D}}{p^{n_{i}}} x^{i}\right. & \\
& \left.a_{i}, b_{i} \in \mathbb{Z},\left(a_{i}, b_{i}, p\right)=1 \text { and }\left|a_{i}\right|,\left|b_{i}\right|<p^{2 M / 3}\right\} \text { modulo } p^{M} \mathcal{O}_{K_{p}},
\end{aligned}
$$

where

$$
\widetilde{P}_{D}(x):=\prod_{C \in I_{K}(f) / P_{K, 1}(f \infty)}\left(x-u(C)^{1 / n_{D}}\right) \in K_{p}[x] .
$$

The factor 2 which multiplies $\widetilde{P}_{D}(x)$ in (5.7) comes from the fact that $\mathcal{O}_{K}=$ $\mathbb{Z}\left[\frac{1+\sqrt{D}}{2}\right]$.

We will compute the $p$-adic invariant $u(C)=u(r, \tau)$ in three steps. The completion $K_{p}$ is the unique quadratic unramified extension of $\mathbb{Q}_{p}$. Let $\log _{p}: K_{p}^{\times} \rightarrow \mathcal{O}_{K_{p}}$ denote the branch of the $p$-adic logarithm which vanishes on $p$. Let $\zeta$ be a primitive $\left(p^{2}-1\right)$-th root of unity in $K_{p}^{\times}$and $\operatorname{let} \log _{\zeta}$ denote the discrete logarithm with base $\zeta$ :

$$
\log _{\zeta}: K_{p}^{\times} \rightarrow \mathbb{Z} /\left(p^{2}-1\right) \mathbb{Z}
$$


where $\frac{x}{p^{\text {ord } p(x)} \zeta^{\log _{\zeta}(x)}} \in 1+p \mathcal{O}_{K_{p}}$ for all $x \in K_{p}^{\times}$. For odd $p$ we have a decomposition

$$
K_{p}^{\times} \simeq \mathbb{Z} \times \mathbb{Z} /\left(p^{2}-1\right) \mathbb{Z} \times p \mathcal{O}_{K_{p}}
$$

given by $x \mapsto\left(\operatorname{ord}_{p}(x), \log _{\zeta}(x), \log _{p}(x)\right)$.

Using the assumption that $\tau$ is reduced we see that the computation of $u(r, \tau)$ boils down to the computation of the following three quantities:

$$
\begin{aligned}
\operatorname{ord}_{p}(u(r, \tau)) & =\psi_{r}\left(\infty \rightarrow \gamma_{\tau} \infty\right), \\
\log _{\zeta} u(r, \tau) & =\int_{\mathbb{X}} \log _{\zeta}(x-\tau y) d \widetilde{\mu}_{r}\left\{\infty \rightarrow \gamma_{\tau} \infty\right\}(x, y), \\
\log _{p} u(r, \tau) & =\int_{\mathbb{X}} \log _{p}(x-\tau y) d \widetilde{\mu}_{r}\left\{\infty \rightarrow \gamma_{\tau} \infty\right\}(x, y) .
\end{aligned}
$$

5.1. Computation of $\operatorname{ord}_{p}(u(r, \tau))$. Let $N$ be an arbitrary integer and let $\left\{g_{1}, \ldots, g_{r}\right\}$ be a finite set of generators of $\Gamma_{0}(N)$. Any element $g \in G$ can be written as a reduced word $g=w_{1} w_{2} \ldots w_{n}$ where $w_{i} \in\left\{g_{1}, g_{1}^{-1}, \ldots, g_{r}, g_{r}^{-1}\right\}$ and $w_{i} \neq x_{i+1}^{-1}$ for all $1 \leq i \leq n-1$. For any integer $k \geq 1$ we let $W_{k}=\prod_{i=1}^{k} w_{i}$. A direct computation reveals that

$$
[\infty]-[g(\infty)]=\sum_{i=1}^{n} W_{n-i}\left([\infty]-\left[w_{n-i+1}(\infty)\right]\right) .
$$

Let $\mathcal{M}=\operatorname{Div}_{0}\left(\Gamma_{0}(N)\{\infty\}\right)$, endowed with its natural left $\Gamma_{0}(N)$-action. Then the next proposition is essential for the explicit computation of $u(r, \tau)$.

Proposition 5.1. The module $\mathcal{M}$ is generated by the elements $\left\{[\infty]-\left[g_{i} \infty\right]\right\}_{i=1}^{r}$ over the ring $\mathbb{Z}\left[\Gamma_{0}(N)\right]$.

Proof. This follows directly from (5.8). Note that if $w_{i}=g_{j}^{-1}$, then

$$
\left([\infty]-\left[g_{j}^{-1}(\infty)\right]\right)=-g_{j}^{-1}\left([\infty]-\left[g_{j}(\infty)\right]\right) .
$$

From now on assume that the prime $p \equiv 1(\bmod 3)$ is fixed. Recall that $f=3$ and $N_{0}=4$. The group $\Gamma_{0}\left(f N_{0}\right)=\Gamma_{0}(12)$ can be generated by the following matrices:

$$
\begin{gathered}
g_{1}=\left(\begin{array}{ll}
1 & 1 \\
0 & 1
\end{array}\right), \quad g_{2}=\left(\begin{array}{cc}
5 & -1 \\
36 & -7
\end{array}\right), \quad g_{3}=\left(\begin{array}{cc}
5 & -4 \\
24 & -19
\end{array}\right), \\
g_{4}=\left(\begin{array}{cc}
7 & -5 \\
24 & -17
\end{array}\right), \quad g_{5}=\left(\begin{array}{cc}
5 & -3 \\
12 & -7
\end{array}\right) .
\end{gathered}
$$

Let $j \in(\mathbb{Z} / f \mathbb{Z})^{\times} /\langle\bar{p}\rangle \simeq(\mathbb{Z} / 3 \mathbb{Z})^{\times}$and $\gamma \in \Gamma_{0}\left(f N_{0}\right)$. We define the period

$$
\pi_{j}(\gamma):=\psi_{j}\{\infty \rightarrow \gamma \infty\}=\frac{1}{2 \pi i} \int_{\infty}^{\gamma \infty} \widetilde{F}_{2}(j, z) d z \in \mathbb{Z}
$$

A computation shows that $\pi_{j}(\gamma)$ satisfies the cocycle condition,

$$
\pi_{j}\left(\gamma_{1} \gamma_{2}\right)=\pi_{j}\left(\gamma_{1}\right)+\pi_{\gamma_{1}^{-1} \star j}\left(\gamma_{2}\right),
$$

for all $\gamma_{1}, \gamma_{2} \in \Gamma_{0}\left(f N_{0}\right)$. A direct computation shows that $D_{1,1}^{j(\bmod f)}(a, c)=$ $D_{1,1}^{-j}(\bmod f)(a, c)$ (this is true for any $\left.f\right)$. Therefore, from the explicit formula given 
in Proposition 4.3 for $\pi_{j}(\gamma)$, we deduce that $\pi_{j}(\gamma)=\pi_{-j}(\gamma)$ for any $\gamma \in \Gamma_{0}\left(f N_{0}\right)$. Now using the previous observation in (5.9), with $f=3$, we deduce that

$$
\pi_{j}\left(\gamma_{1} \gamma_{2}\right)=\pi_{j}\left(\gamma_{1}\right)+\pi_{j}\left(\gamma_{2}\right)
$$

Let $\gamma=\left(\begin{array}{ll}a & b \\ c & d\end{array}\right) \in \Gamma_{0}\left(f N_{0}\right)$. Then in general if the height of $\gamma \infty=\frac{a}{c}$ is large, the direct computation of the period $\pi_{j}(\gamma)$ via the explicit formula given in Proposition 4.3 tends to be long since the summation of the corresponding Dedekind sum depends linearly on $c$. Instead we compute once and for all the four periods

$$
\left\{\pi_{1}\left(g_{i}\right)\right\}_{i=2}^{5} .
$$

Note that trivially one has $\pi_{1}\left(g_{1}\right)=0$. Now using the command "FindWord"in Magma, one can obtain an expression of the form $\gamma=w_{1} w_{2} \ldots w_{n}$ where the $w_{j}$ 's are elements in the set $\left\{g_{j}^{ \pm 1}\right\}_{j=1}^{5}$. From (5.10) it follows that

$$
\pi_{1}(\gamma)=\sum_{i=1}^{n} \pi_{1}\left(w_{i}\right)
$$

Note that $\pi_{1}\left(g_{i}^{-1}\right)=-\pi_{1}\left(g_{i}\right)$. We have thus succeded to compute the period $\pi_{1}(\gamma)$ purely in terms of the periods $\left\{\pi_{1}\left(g_{i}\right)\right\}_{i=2}^{5}$. In particular, this method gives us a quick way of computing $\pi_{1}\left(\gamma_{\tau}\right)$ where $\gamma_{\tau}$ is the matrix appearing in (5.5)).

5.2. Computation of $\log _{\zeta} u(r, \tau)$. For $m=\left[c_{1}\right]-\left[c_{2}\right] \in \mathcal{M}=\operatorname{Div}_{0}\left(\Gamma_{0}\left(f N_{0}\right)\{\infty\}\right)$, let $\widetilde{\mu}_{r}[m]:=\widetilde{\mu}_{r}\left\{c_{1} \rightarrow c_{2}\right\}$. Since an arbitrary $m \in \mathcal{M}$ can be written as a sum of elements of the form $\left[c_{1}\right]-\left[c_{2}\right]$ we may define $\widetilde{\mu}_{r}[m]$ by linearity. If $\tau \in \mathcal{H}_{p}$ and $\gamma \in \Gamma_{0}\left(f N_{0}\right)$, then a formal computation shows that

$$
\int_{\mathbb{X}} \log _{\zeta}(x-\tau y) d \widetilde{\mu}_{r}[\gamma m](x, y)=\int_{\mathbb{X}} \log _{\zeta}\left(x-y \gamma^{-1} \tau\right) d \widetilde{\mu}_{\gamma^{-1} \star r}[m](x, y) .
$$

The last equality uses the fact that the total measure of $\widetilde{\mu}_{\gamma^{-1} \star r}[\mathrm{~m}]$ is zero and that for all compact open sets $U \in \mathbb{X}$ one has that $\widetilde{\mu}_{r}[m](U)=\widetilde{\mu}_{\gamma^{-1} \star r}\left[\gamma^{-1} m\right]\left(\gamma^{-1} U\right)$. Now let $\gamma \in \Gamma_{0}\left(f N_{0}\right)$ and let $\gamma=w_{1} w_{2} \ldots w_{n}$ where $w_{i} \in\left\{g_{j}^{ \pm 1}\right\}_{j=1}^{5}$. From (5.8) we deduce that

$$
[\infty]-[\gamma \infty]=\sum_{i=1}^{n} W_{n-i}\left([\infty]-\left[w_{n-i+1}(\infty)\right]\right),
$$

where $W_{k}=\prod_{j=1}^{k} w_{j}$. If $w_{n-i+1}=g_{j}$ for some $j$, then we deduce from (5.12) that

$$
\begin{aligned}
& \int_{\mathbb{X}} \log _{\zeta}(x-\tau y) d \widetilde{\mu}_{r}\left[W_{n-i}\left([\infty]-\left[w_{n-i+1}\right]\right)\right](x, y) \\
& =\int_{\mathbb{X}} \log _{\zeta}\left(x-y W_{n-i}^{-1} \tau\right) d \widetilde{\mu}_{W_{n-i}^{-1} \star r}\left\{\infty \rightarrow g_{j} \infty\right\}(x, y) .
\end{aligned}
$$

If $w_{n-i+1}=g_{j}^{-1}$ for some $j$, then we obtain in a similar way that

$$
\begin{aligned}
& \int_{\mathbb{X}} \log _{\zeta}(x-\tau y) d \widetilde{\mu}_{r}\left[W_{n-i}\left([\infty]-\left[w_{n-i+1}\right]\right)\right](x, y) \\
& =\int_{\mathbb{X}} \log _{\zeta}\left(x-y W_{n-i}^{-1} \tau\right) d \widetilde{\mu}_{W_{n-i}^{-1} \star r}\left\{\infty \rightarrow g_{j}^{-1} \infty\right\}(x, y) \\
& =-\int_{\mathbb{X}} \log _{\zeta}\left(x-y\left(W_{n-i} g_{j}\right)^{-1} \tau\right) d \widetilde{\mu}_{\left(W_{n-i} g_{j}\right)^{-1} \star r}\left\{\infty \rightarrow g_{j} \infty\right\}(x, y) .
\end{aligned}
$$


We thus see that in order to compute

$$
\int_{\mathbb{X}} \log _{\zeta}(x-\tau y) d \widetilde{\mu}_{r}\{\infty \rightarrow \gamma \infty\}(x, y),
$$

it is sufficient to compute

$$
\int_{\mathbb{X}} \log _{\zeta}\left(x-\tau^{\prime} y\right) d \widetilde{\mu}_{r^{\prime}}\left\{\infty \rightarrow g_{j} \infty\right\}(x, y),
$$

for certain $\tau^{\prime} \in \mathcal{H}, r^{\prime} \in(\mathbb{Z} / 3 \mathbb{Z})^{\times}$and $j \in\{2,3,4,5\}$ which depend on the word representing $\gamma$. To compute (5.13) it is enough to take a cover of $\mathbb{X}$ in which $x$ and $y$ are determined modulo $p$. Therefore, it is enough to compute

$$
\sum_{\substack{0 \leq u, v \leq p-1 \\(u, v) \neq(0,0)}} \log _{\zeta}\left(u-\tau^{\prime} v\right) \widetilde{\mu}_{r^{\prime}}\left\{\infty \rightarrow g_{j} \infty\right\}\left(\left(u+p \mathbb{Z}_{p}\right) \times\left(v+p \mathbb{Z}_{p}\right)\right) \in \mathbb{Z} /\left(p^{2}-1\right) \mathbb{Z} .
$$

This can be efficiently computed using the explicit formulas for the $\widetilde{\mu}_{r^{\prime}}\left\{\infty \rightarrow g_{j} \infty\right\}$ measure of the balls $\left(u+p \mathbb{Z}_{p}\right) \times\left(v+p \mathbb{Z}_{p}\right)$ given in Proposition 4.1.

5.3. Computation of $\log _{p} u(r, \tau)$. This subsection mirrors pages 12 and 13 of Das07a. We included it only for the reader's convenience. By arguments analogous to those of Section 5.2 and replacing $\log _{\zeta}$ by $\log _{p}$ we see that in order to compute

$$
\int_{\mathbb{X}} \log _{p}(x-\tau y) d \widetilde{\mu}_{r}\{\infty \rightarrow \gamma \infty\}(x, y),
$$

it is sufficient to compute

$$
\int_{\mathbb{X}} \log _{p}\left(x-\tau^{\prime} y\right) d \widetilde{\mu}_{r^{\prime}}\left\{\infty \rightarrow g_{j} \infty\right\}(x, y)
$$

for certain $\tau^{\prime} \in \mathcal{H}_{p}, r^{\prime} \in(\mathbb{Z} / 3 \mathbb{Z})^{\times}$and $j \in\{2,3,4,5\}$ which depend on the word representing $\gamma$. In order to compute (5.15) we will use the same method that was developed in Das07a.

The integral (5.15) can be rewritten as follows:

$$
\begin{aligned}
\int_{\mathbb{X}} \log _{p}\left(x-y \tau^{\prime}\right) d \widetilde{\mu}_{r^{\prime}}\left\{\infty \rightarrow g_{j} \infty\right\}(x, y) \\
=\int_{\mathbb{Z}_{p} \times \mathbb{Z}_{p}^{\times}} \log _{p}\left(x-y \tau^{\prime}\right) d \widetilde{\mu}_{r^{\prime}}\left\{\infty \rightarrow g_{j} \infty\right\}(x, y) \\
\quad+\int_{\mathbb{Z}_{p}^{\times} \times p \mathbb{Z}_{p}} \log _{p}\left(x-y \tau^{\prime}\right) d \widetilde{\mu}_{r^{\prime}}\left\{\infty \rightarrow g_{j} \infty\right\}(x, y) \\
=\int_{\mathbb{Z}_{p} \times \mathbb{Z}_{p}^{\times}} \log _{p}(y) d \widetilde{\mu}_{r^{\prime}}\left\{\infty \rightarrow g_{j} \infty\right\}(x, y) \\
\quad+\int_{\mathbb{Z}_{p}^{\times} \times p \mathbb{Z}_{p}} \log _{p}(x) d \widetilde{\mu}_{r^{\prime}}\left\{\infty \rightarrow g_{j} \infty\right\}(x, y) \\
\quad+\int_{\mathbb{Z}_{p} \times \mathbb{Z}_{p}^{\times}} \log _{p}\left(\frac{x}{y}-\tau^{\prime}\right) d \widetilde{\mu}_{r^{\prime}}\left\{\infty \rightarrow g_{j} \infty\right\}(x, y) \\
\quad+\int_{\mathbb{Z}_{p}^{\times} \times p \mathbb{Z}_{p}} \log _{p}\left(1-\tau^{\prime} \frac{y}{x}\right) d \widetilde{\mu}_{r^{\prime}}\left\{\infty \rightarrow g_{j} \infty\right\}(x, y) .
\end{aligned}
$$


Suppose we want to calculate (5.16) to an accuracy of $M p$-adic digits. First observe that the first two terms of (5.16) are independent of $\tau^{\prime}$. To evaluate the first term, one finds a polynomial $f(y) \in \mathbb{Q}[y]$ such that $\left|f(y)-\log _{p}(y)\right|_{p}<\frac{1}{p^{M+1}}$ for all $y \in \mathbb{Z}_{p}^{\times}$. To construct $f(y)$ consider the polynomial

$$
g_{i}(y)=\prod_{\substack{j=1 \\ j \neq i}}^{p-1}(y-j)^{M} .
$$

Let $h_{i}(y)$ denote the power series expansion of $\log _{p}(y) / g_{i}(y)$ on the residue disc $i+p \mathbb{Z}_{p}$, truncated at $M+[\log M]$ terms, where $[\log M]$ denotes the integer part of $M$. In order to compute this truncated power series one can compute the Taylor series expansions around $y_{0}=0$ of $\log _{p}(y+i)=\log _{p} i+\log _{p}\left(1+\frac{y}{i}\right)$ and $\frac{1}{g_{i}(y+i)}$ up to order $M+[\log M]$, multiply them and finally apply the change of variables $y \mapsto y-i$. Letting

$$
f(y)=\sum_{i=1}^{p-1} g_{i}(y) h_{i}(y),
$$

we obtain the required polynomial which has degree $(p-1) M+[\log M]$. The first term of (5.16) may be evaluated by replacing $\log _{p} y$ by $f(y)$. Then if $y^{n}$ is a monomial of $f(y)$ we can use Propositon 4.4 which gives an explicit formula for the integral of $y^{n}$ on $\mathbb{Z}_{p} \times \mathbb{Z}_{p}^{\times}$against the measure $\mu_{r^{\prime}}\left\{\infty \rightarrow g_{j} \infty\right\}$.

To compute the second term of (5.16) up to an accuracy of $M p$-adic digits it is enough to compute

$$
\int_{\mathbb{Z}_{p}^{\times} \times p \mathbb{Z}_{p}} f(x) d \widetilde{\mu}_{r^{\prime}}\left\{\infty \rightarrow g_{j} \infty\right\}(x, y) .
$$

Taking a monomial $x^{n}$ of $f(x)$, we see that in order to compute (5.18) it is sufficient to compute the integral

$$
\begin{aligned}
\int_{\mathbb{Z}_{p}^{\times} \times p \mathbb{Z}_{p}} x^{n} d \widetilde{\mu}_{r^{\prime}}\left\{\infty \rightarrow g_{j} \infty\right\}(x, y)= & \int_{\mathbb{X}} x^{n} d \widetilde{\mu}_{r^{\prime}}\left\{\infty \rightarrow g_{j} \infty\right\}(x, y) \\
& -\int_{p \mathbb{Z}_{p} \times \mathbb{Z}_{p}^{\times}} x^{n} d \widetilde{\mu}_{r^{\prime}}\left\{\infty \rightarrow g_{j} \infty\right\}(x, y) .
\end{aligned}
$$

Applying Propositions 4.2 and 4.4 to the right hand-side of this equality we deduce that

$$
\begin{aligned}
\int_{\mathbb{Z}_{p}^{\times} \times p \mathbb{Z}_{p}} x^{n} d \widetilde{\mu}_{r^{\prime}}\left\{\infty \rightarrow g_{j} \infty\right\}(x, y)=\sum_{l=0}^{n}\left(\begin{array}{c}
n \\
l
\end{array}\right)\left(\frac{a}{c}\right)^{n-l}(-1)^{l} \\
\cdot \sum_{d_{0} \mid N_{0}, r^{\prime} \in \mathbb{Z} / f \mathbb{Z}} n\left(d_{0}, r^{\prime}\right) d_{0}^{-l}\left(p^{n} D_{n-l+1, l+1}^{j r^{\prime}(\bmod f)}\left(a, c / d_{0}\right)\right. \\
\left.-p^{n-l} D_{n-l+1, l+1}^{j r^{\prime}(\bmod f)}\left(p a, c / d_{0}\right)\right) .
\end{aligned}
$$

This completes the evaluation for the second term of (5.16).

The third term of (5.16) can be evaluated in the following way. We have a natural projection map $\pi: \mathbb{X} \rightarrow \mathbb{P}^{1}\left(\mathbb{Q}_{p}\right)$ given by $\pi(x, y)=\frac{x}{y}$. From Lemma 6.1 of 
Cha one has $\pi_{*} \widetilde{u}_{r^{\prime}}\left\{c_{1} \rightarrow c_{2}\right\}=\mu_{r^{\prime}}\left\{c_{1} \rightarrow c_{2}\right\}$ where $\mu_{r^{\prime}}\left\{c_{1} \rightarrow c_{2}\right\}$ is the $\mathbb{Z}$-valued measure on $\mathbb{P}^{1}\left(\mathbb{Q}_{p}\right)$ appearing in Definiton 5.2 of [Cha]. Therefore, we have

$$
\begin{gathered}
\int_{\mathbb{Z}_{p} \times \mathbb{Z}_{p}^{\times}} \log _{p}\left(\frac{x}{y}-\tau^{\prime}\right) d \widetilde{\mu}_{r^{\prime}}\left\{\infty \rightarrow g_{j} \infty\right\}(x, y) \\
=\int_{\mathbb{Z}_{p}} \log _{p}\left(t-\tau^{\prime}\right) d \mu_{r^{\prime}}\left\{\infty \rightarrow g_{j} \infty\right\}(t) .
\end{gathered}
$$

We have

$$
\begin{aligned}
\int_{\mathbb{Z}_{p}} \log _{p}\left(t-\tau^{\prime}\right) d \mu_{r^{\prime}}\left\{\infty \rightarrow g_{j} \infty\right\}(t) \\
\quad=\sum_{i=0}^{p-1} \int_{i+p \mathbb{Z}_{p}} \log _{p}(t-i+(i-\tau)) d \mu_{r^{\prime}}\left\{\infty \rightarrow g_{j} \infty\right\} \\
=\sum_{i=0}^{p-1}\left[\log _{p}\left(\tau^{\prime}-i\right) \mu_{r^{\prime}}\left(i+p \mathbb{Z}_{p}\right)+\int_{i+p \mathbb{Z}_{p}} \log _{p}\left(1+\frac{t-i}{\tau^{\prime}-i}\right) d \mu_{r^{\prime}}(t)\right]
\end{aligned}
$$

The integrand in (5.20) can be written as a power series in each residue disc $i+p \mathbb{Z}_{p}$. Therefore, in order to calculate the integral modulo $p^{M}$ it is enough to calculate the moments

$$
\begin{aligned}
\int_{i+p \mathbb{Z}_{p}} & (t-i)^{n} d \mu_{r^{\prime}}\left\{\infty \rightarrow g_{j} \infty\right\} \\
= & p^{n} \int_{\mathbb{Z}_{p}} u^{n} d \mu_{r^{\prime}}\left\{\infty \rightarrow \frac{e}{e p^{m}}\right\}(u) \quad\left(\bmod p^{M}\right)
\end{aligned}
$$

for $n=0, \ldots, M-1$ where $P_{i}=\left(\begin{array}{cc}p & i \\ 0 & 1\end{array}\right)$ and $P_{i}^{-1} g_{j} \infty=\frac{b}{e p^{m}}$ with $(e, p)=1$. (The equality (5.21) uses the invariance of $\mu_{r^{\prime}}$ under $P_{i} \in \widetilde{\Gamma}_{0}$.) If we pull back (5.21) to $\mathbb{X}$, we get

$$
\begin{aligned}
\int_{\mathbb{Z}_{p}} u^{n} d \mu_{r^{\prime}}\left\{\infty \rightarrow \frac{b}{e p^{m}}\right\}=\int_{\mathbb{Z}_{p} \times \mathbb{Z}_{p}^{\times}} x^{n} y^{-n} d \mu_{r^{\prime}}\{\infty \rightarrow w\}(x, y) \\
=\lim _{\substack{j \rightarrow \infty \\
g_{j}=(p-1) p^{j}}} \int_{\mathbb{Z}_{p} \times \mathbb{Z}_{p}^{\times}} x^{n} y^{g-n} d \mu_{r^{\prime}}\left\{\infty \rightarrow \frac{b}{e p^{m}}\right\}(x, y) \\
=-\lim _{j \rightarrow \infty} \frac{12}{f^{n}} \sum_{l=0}^{n}\left(\begin{array}{c}
n \\
l
\end{array}\right)\left(\frac{a}{c}\right)^{n-l}(-1)^{l} \\
\quad \sum_{d_{0} \mid N_{0}, r \in(\mathbb{Z} / f \mathbb{Z})^{\times}} n\left(d_{0}, r\right) d_{0}^{-l} D_{g_{j}-l+1, l+1}^{j r^{\prime}(\bmod f)}\left(b, e p^{m} / d_{0}\right) .
\end{aligned}
$$


Let us fix a value $r^{\prime} \in(\mathbb{Z} / f \mathbb{Z})^{\times}$and let us assume that $m \geq 1$. The expression (5.22) can be written in terms of the single-variable measures appearing in Definition 3.3 .

$$
\begin{aligned}
\lim _{j \rightarrow \infty} & \sum_{d_{0} \mid N_{0} \in(\mathbb{Z} / f \mathbb{Z})^{\times}} n\left(d_{0}, r^{\prime}\right) d_{0}^{-l} D_{g_{j}-l+1, l+1}^{j r^{\prime}(\bmod f)}\left(b, e p^{m} / d_{0}\right) \\
= & \sum_{h=1}^{e p^{m}} \frac{\widetilde{B}_{l+1}\left(\frac{h b}{e p^{m}}\right)}{l+1} \lim _{j \rightarrow \infty} \mathcal{F}_{g_{j}-l+1}\left(h+e p^{m} Z\right) \\
= & \sum_{\substack{h=1 \\
(h, p)=1}}^{e p^{m}} \frac{\widetilde{B}_{l+1}\left(\frac{h b}{e p^{m}}\right)}{l+1} \int_{h+e p^{m} Z} x_{p}^{-l} d \mathcal{F}_{1}(x) .
\end{aligned}
$$

The second equality comes from the observation that, when $p \mid h$,

$$
\lim _{j \rightarrow \infty} \mathcal{F}_{g_{j}-l+1}\left(h+e p^{m} Z\right)=\lim _{j \rightarrow \infty} p^{g_{j}-l+1} \mathcal{F}_{g_{j}-l+1}\left(h / p+e p^{m-1} Z\right)=0,
$$

where the middle equality of (5.23) follows from (3.6). Note that when $(h, p)=1$, the function $x \mapsto x_{p}^{-l}$ is continuous on the ball $h+e p^{m} Z$ and therefore the integral $\int_{h+e p^{m} Z} x_{p}^{-l} d \mathcal{F}_{1}(x)$ makes sense. In the case where $m \geq 1$ and $(h, p)=1$, one can compute $\int_{h+e p^{m} Z} x_{p}^{-l} d \mathcal{F}_{1}(x)$ by expanding the function $x_{p}^{-l}$ in a neighboorhood of $h+p \mathbb{Z}_{p}$. We consider the Taylor series expansion

$$
\begin{aligned}
x_{p}^{-l} & =h^{-l}\left(1+\left(\frac{x_{p}-h}{h}\right)\right)^{-l} \\
& =h^{-l} \sum_{j=0}^{M}\left(\frac{x_{p}-h}{h}\right)^{j}+\left(\frac{x_{p}-h}{h}\right)^{M+1} H\left(x_{p}\right),
\end{aligned}
$$

where $x \mapsto H\left(x_{p}\right)$ is some continuous function on $h+e p^{m} Z$. From (5.24) we deduce that

$$
\int_{h+e p^{m} Z} x_{p}^{-l} d \mathcal{F}_{1}(x) \equiv h^{-l} \int_{h+e p^{m} Z} \sum_{j=0}^{M}\left(\frac{x_{p}-h}{h}\right)^{j} d \mathcal{F}_{1}(x) \quad\left(\bmod p^{M}\right) .
$$

Now expanding the finite sum $\sum_{j=0}^{M}\left(\frac{x_{p}-h}{h}\right)^{j}$ as a polynomial in $x_{p}$ and using Proposition 3.1 together with (3.5), we get an approximation to $\int_{h+e p^{m} Z} x_{p}^{-l} d \mathcal{F}_{1}(x)$ up to a precision of $M p$-adic digits.

In the case where $m=0$ one can assume, without loss of generality, that $(h, p)=$ 1. (Otherwise replace $h$ by $h+e$ and observe that $(h+e, p)=1$ and $h+e Z=$ $(h+e)+e Z)$. We define

$$
\int_{h+e Z} x_{p}^{-l} d \mathcal{F}_{1}(x):=\sum_{\substack{1 \leq h^{\prime} \leq p e \\\left(h^{\prime}, p\right)=1 \\ h^{\prime} \equiv h(\bmod e)}} \int_{h^{\prime}+e p Z} x_{p}^{-l} d \mathcal{F}_{1}(x) .
$$


Note that one cannot integrate directly $x_{p}^{-l}$ against $\mathcal{F}_{1}$ on the compact open set $h+e Z$ since the function $x_{p}^{-l}$ (for $l \geq 1$ ) is not continuous on $h+e Z$. Using the definition above and (5.25) one obtains an approximation to $\int_{h+e Z} x_{p}^{-l} d \mathcal{F}_{1}(x)$ to $M p$-adic digits. This concludes the explicit calculation of the third term of (5.16).

The fourth term of (5.16) can be evaluated in the following way. First note that

$$
\begin{aligned}
& \int_{\mathbb{Z}_{p}^{\times} \times p \mathbb{Z}_{p}} \log _{p}\left(1-\tau^{\prime} \frac{y}{x}\right) d \widetilde{\mu}_{r^{\prime}}\left\{\infty \rightarrow g_{j} \infty\right\}(x, y) \\
& =\int_{\mathbb{P}^{1}\left(\mathbb{Q}_{p}\right) \backslash \mathbb{Z}_{p}} \log _{p}\left(1-\frac{\tau^{\prime}}{t}\right) d \mu_{r^{\prime}}\left\{\infty \rightarrow g_{j} \infty\right\}(t) .
\end{aligned}
$$

Now using the Taylor series expansion

$$
-\log _{p}\left(1-\frac{\tau^{\prime}}{t}\right)=\sum_{j=1}^{\infty} \frac{\tau^{\prime n}}{t^{n}}
$$

which is valid for any $t \in \mathbb{P}^{1}\left(\mathbb{Q}_{p}\right) \backslash \mathbb{Z}_{p}$, we see that in order to compute (5.26) to an accuracy of $M p$-adic digits it is sufficient to compute the moments

$$
\int_{\mathbb{P}^{1}\left(\mathbb{Q}_{p}\right) \backslash \mathbb{Z}_{p}} t^{-n} d \mu_{r^{\prime}}\left\{\infty \rightarrow g_{j} \infty\right\}(t),
$$

for $0 \leq n \leq M$, to a precision of $M p$-adic digits. Let $g_{j} \infty=\frac{a_{j}}{c_{j}}$. The invariance of $\mu_{r^{\prime}}\left\{\infty \rightarrow \frac{a_{j}}{c_{j}}\right\}$ under the matrix $\gamma=\left(\begin{array}{cc}1 & 0 \\ f N_{0} & 1\end{array}\right)$ implies that

$$
\begin{aligned}
& \int_{\mathbb{P}^{1}\left(\mathbb{Q}_{p}\right) \backslash \mathbb{Z}_{p}} t^{-n} d \mu_{r^{\prime}}\left\{\infty \rightarrow \frac{a_{j}}{c_{j}}\right\}(t) \\
& =-\int_{\frac{1}{f N_{0}}+p \mathbb{Z}_{p}}\left(\frac{u}{-f N_{0} u+1}\right)^{-n} d \mu_{\gamma^{-1} \star r^{\prime}}\left\{\infty \rightarrow-\frac{1}{f N_{0}}\right\}(u) \\
& \quad+\int_{\frac{1}{f N_{0}}+p \mathbb{Z}_{p}}\left(\frac{u}{-f N_{0} u+1}\right)^{-n} d \mu_{\gamma^{-1} \star r^{\prime}}\left\{\infty \rightarrow \frac{a_{j}}{-f N_{0} a_{j}+c_{j}}\right\}(u) .
\end{aligned}
$$

Let $j$ be the positive integer less than $p$ which is congruent to $\frac{1}{f N_{0}}$ modulo $p$. The function $\left(\frac{u}{-f N_{0} u+1}\right)^{-n}$ can be expanded as a power series in $u-j$ on the residue disc $j+p \mathbb{Z}_{p}$. This reduces the computation of (5.28) to that of integrals of the form

$$
\int_{j+p \mathbb{Z}_{p}}(u-j)^{n} d \mu_{r^{\prime}}\{\infty \rightarrow w\}(u)
$$

for $0 \leq n \leq M, r \in(\mathbb{Z} / f \mathbb{Z})^{\times}$and $w=-\frac{1}{f N_{0}}$ or $\frac{a_{j}}{-f N_{0} a_{j}+c_{j}}$. Note that (5.29) is an expression with the same shape as the left-hand side of (5.21). This concludes the explicit computation of the fourth term of (5.16). 


\section{The ALGORITHM}

We have thus reduced the computation of

$$
u(r, \tau)=p^{\psi_{j}\left\{\infty \rightarrow \gamma_{\tau} \infty\right\}} \oint_{\mathbb{X}}(x-\tau y) d \widetilde{\mu}_{r}\left\{\infty \rightarrow \gamma_{\tau} \infty\right\}(x, y),
$$

up to an accuracy of $M p$-adic digits, to the computation of the following quantities:

\section{Part 1 of the program (independent of $D$ ).}

(1) We compute exactly the set of Bernoulli numbers $B_{n}$ for $0 \leq n \leq$ $(p-1) M+[\log M]$ and store these in a file. The use of this data and the explicit polynomial expression (3.2) for $B_{n}(x)$, allow us to construct Bernoulli polynomials more efficiently since all the $B_{n}$ 's are only computed once.

(2) For $j \in\{2,3,4,5\}$ and $i \in\{0,1, \ldots, p-1\}$, we compute $\mu_{1}\left\{\infty \rightarrow g_{j} \infty\right\}\left(\mathbb{Z}_{p}\right)$ and $\mu_{1}\left\{\infty \rightarrow M_{i} g_{j} \infty\right\}\left(\mathbb{Z}_{p}\right)$, where $M_{i}:=\left(\begin{array}{cc}1 & -i \\ 0 & p\end{array}\right)$. Here we use the explicit formula of Proposition 4.3 .

(3) For $r \in(\mathbb{Z} / 3 \mathbb{Z})^{\times}, j \in\{2,3,4,5\}$ and $0 \leq u, v \leq p-1$ such that $(u, v) \neq$ $(0,0)$, we compute $\widetilde{\mu}_{r}\left\{\infty \rightarrow g_{j} \infty\right\}\left(\left(u+p \mathbb{Z}_{p}\right) \times\left(v+p \mathbb{Z}_{p}\right)\right)$ using the explicit formulas of Proposition 4.1 .

(4) For $r \in(\mathbb{Z} / 3 \mathbb{Z})^{\times}, j \in\{2,3,4,5\}$, we compute $\int_{\mathbb{Z}_{p} \times \mathbb{Z}_{p}^{\times}} f(y) d \mu_{r}\left\{\infty \rightarrow g_{j} \infty\right\}$ where $f(y)$ is the polynomial in $y$ appearing in (5.17). Here we use the explicit formulas of Proposition 4.4

(5) For $r \in(\mathbb{Z} / 3 \mathbb{Z})^{\times}, j \in\{2,3,4,5\}$ we compute $\int_{p \mathbb{Z}_{p} \times \mathbb{Z}_{p}^{\times}} f(x) d \mu_{r}\left\{\infty \rightarrow g_{j} \infty\right\}$ using (5.19).

(6) Finally, for $0 \leq i \leq p-1,0 \leq n \leq M, r \in(\mathbb{Z} / 3 \mathbb{Z})^{\times}$and

$$
\begin{aligned}
& w \in\left\{g_{2} \infty, g_{3} \infty, g_{4} \infty, g_{5} \infty, \gamma^{-1} g_{2} \infty, \gamma^{-1} g_{3} \infty, \gamma^{-1} g_{4} \infty, \gamma^{-1} g_{5} \infty,-\frac{1}{12}\right\} \\
& \text { where } \gamma=\left(\begin{array}{cc}
1 & 0 \\
12 & 1
\end{array}\right) \text {, we compute } \int_{i+p \mathbb{Z}_{p}}(t-i)^{n} d \mu_{r}\{\infty \rightarrow w\}(t) \text { using } \\
& \quad \text { (5.21). }
\end{aligned}
$$

Note that the quantities appearing in (1), (2), (3), (4), (5) and (6) do not depend on $D$ and $\tau$. Therefore, one only needs to compute them once. The computation of the quantities (4), (5) and (6) are the ones which contribute the most to the running time of the algorithm. We store all these quantities in various files.

Part 2 of the program (depends on $D$ ). Let $D$ be an admissible discriminant and let $K=\mathbb{Q}(\sqrt{D})$. We now want to explain how to compute the polynomials $\widetilde{P}_{D}(x)$. Let $\mathfrak{c}=(1+f \sqrt{D}) \mathcal{O}_{K}$. Assume that one has a complete set of pairs $\left\{\left(r_{i}, \tau_{i}\right)\right\}_{i=1}^{h}$ such that the ideals $\left\{r_{i} \Lambda_{\tau_{i}}\right\}_{i=1}^{h}$ form a complete set of representatives $\left\{C_{i}\right\}_{i=1}^{h}$ of $I_{K}(3) /\left\langle P_{K, 1}(3 \infty), \mathfrak{c}\right\rangle$, where $h=\# I_{K}(3) /\left\langle P_{K, 1}(3 \infty), \mathfrak{c}\right\rangle$ and $2 h=\# I_{K}(3) / P_{K, 1}(3 \infty)$. Assume, moreover, that the $\tau_{i}$ 's are chosen in such a way that $\mathcal{O}_{\tau_{i}}=\mathcal{O}_{4 \tau_{i}}=\mathcal{O}_{K}$ and that $\tau_{i}-\tau_{i}^{\sigma}>0$ where $\operatorname{Gal}(K / \mathbb{Q})=\{1, \sigma\}$. For every $i$ one computes $u\left(r_{i}, \tau_{i}\right)$ up to a precision of $M p$-adic digits as explained in Sections 5.1, 5.2 and 5.3, using the outputs produced by part 1 of the program. Then one 
defines

$$
\widetilde{P}_{D}(x)=\prod_{i=1}^{h}\left(x-u\left(r_{i}, \tau_{i}\right)^{\frac{s_{i}}{n_{D}}}\right) \prod_{i=1}^{h}\left(x-u\left(r_{i}, \tau_{i}\right)^{-\frac{s_{i}}{n}}\right),
$$

where $\mathfrak{s}_{i}$ is equal to 1 if $r_{i} \Lambda_{\tau_{i}}$ is equivalent to $C_{i}$ modulo $P_{K, 1}(3 \infty)$ and -1 otherwise. The integer $n_{D}$ is chosen as explained in (5.7). The determination of $n_{D}$ is done empirically and we do not know how to predict it.

Complications related to the choice of the root of unity. The quantity $u(r, \tau)^{1 / n_{D}}$ is only well defined modulo $\mu_{n_{D}}$. In order to compute the polynomial appearing in (6.1) one needs to fix a certain rule in order to specify uniquely the $p$-adic invariant $u\left(r_{i}, \tau_{i}\right)^{1 / n_{D}}$. The computation of $\operatorname{ord}_{p}\left(u\left(r_{i}, \tau_{i}\right)^{1 / n_{D}}\right)$ and $\log _{p}\left(u\left(r_{i}, \tau_{i}\right)^{1 / n_{D}}\right)$ is independent of the choice of this rule but not $\log _{\zeta}\left(u\left(r_{i}, \tau_{i}\right)^{1 / n_{D}}\right)$. In order to specify $\log _{\zeta^{\prime}} u\left(r_{i}, \tau_{i}\right)^{1 / n_{D}}$, where $\zeta^{\prime}$ is a primitive $n_{D}\left(p^{2}-1\right)$-root of unity, we do the following:

(1) We fix a primitive $\left(p^{2}-1\right)$-th root of unity $\zeta \in \mathcal{O}_{K_{p}}^{\times}$.

(2) For every element $x \in \mathcal{O}_{K_{p}}^{\times}$we define $\log _{\zeta} x$ to be the unique integer $n$ such that $0 \leq n \leq p^{2}-2$ and $\zeta^{-n} x \in 1+p \mathcal{O}_{K_{p}}$.

(3) We define $\log _{\zeta} u\left(r_{i}, \tau_{i}\right)$ as a certain integer rather than a residue class of $\mathbb{Z} /\left(p^{2}-1\right) \mathbb{Z}$. In order to say what this integer is, it is enough to interpret the expression appearing in (5.14) as an integer. According to our previous choices (1) and (2), the expression in (5.14) can be viewed as a well-defined integer. Therefore, $\log _{\zeta} u\left(r_{i}, \tau_{i}\right)$ corresponds to a unique integer.

Having fixed this rule, one can then define $u\left(r_{i}, \tau_{i}\right)^{1 / n_{D}}$ as

$$
u\left(r_{i}, \tau_{i}\right)^{\frac{1}{n_{D}}}:=p^{\frac{\operatorname{ord} p\left(u\left(r_{i}, \tau_{i}\right)\right)}{n_{D}}} \zeta^{\frac{\log _{\zeta} u\left(r_{i}, \tau_{i}\right)}{n_{D}}} \exp \left(\frac{\log _{p} u\left(r_{i}, \tau_{i}\right)}{n_{D}}\right) .
$$

In general, a choice of a different rule will give rise to a different polynomial. The reader may compare the tables at the end of Cha with the tables at the end of this paper and notice different answers for the same values of $D, p, f, N_{0}$ and $\delta$. This is accounted for by the fact that we used in Cha a different rule than the one explained above.

Precision needed in order to recognize the coefficients of $\widetilde{P}_{D}(x)$ as elements of $\mathcal{O}_{K}\left[\frac{1}{p}\right]$. Let $\widetilde{P}_{D}(x)=\prod_{i=1}^{2 h}\left(x-u_{i}\right) \in K_{p}[x]$. From our numerical computations, we observed that the number of $p$-adic digits required in order to recognize the coefficients of $\widetilde{P}_{D}(x)$, as elements of $\mathcal{O}_{K}\left[\frac{1}{p}\right]$, is roughly

$$
N=\frac{1}{2} \sum_{i=1}^{2 h}\left|v_{p}\left(u_{i}\right)\right|
$$

The integer $N$ is an upper bound for the largest power of $p$ which can appear in the denominators of the coefficients of $\widetilde{P}(x)$. In all the examples of the tables, this upper bound is attained. 


\section{Discussion of the Results}

The splitting field of the polynomial $\widetilde{P}_{D}(x)$. For simplicity let us assume that $p \equiv 1(\bmod f)$ so that $K(f \infty)^{\langle F r o b(\mathfrak{p} / \wp)\rangle}=K(f \infty)$. In general, one cannot hope that the splitting of $\widetilde{P}_{D}(x)$ generates the full narrow ray class field of $K=\mathbb{Q}(\sqrt{D})$ of conductor $f$. Let us explain an obstruction which is inherent to our construction. Let $u=u(r, \tau)$ be a $p$-unit coming from our $p$-adic construction and let us assume the truth of Conjecture 2.1. Let $\left\{\sigma_{i}: K(u) \hookrightarrow \mathbb{C}\right\}$ be the set of embeddings of $K(u)$ into $\mathbb{C}$. Conjecture 2.1 asserts that $u$ is an algebraic number such that for all $i$,

$$
u^{\sigma_{i}}\left(u^{\sigma_{i}}\right)^{\tau_{\infty}}=1
$$

where $\tau_{\infty}$ is the complex conjugation of $\mathbb{C}$. In particular, if there exists one $\sigma_{i}$ such that $u^{\sigma_{i}} \in \mathbb{R}$, then $u^{\sigma_{i}}= \pm 1$ and therefore $u= \pm 1$. Because of this observation, we will assume from now on that $K(u)$ is a totally complex number field. From (17.1) we deduce that for all $\sigma_{i}$ we have

$$
u^{-1}=u^{\sigma_{i} \tau_{\infty} \sigma_{i}^{-1}} .
$$

In particular, the action of a complex conjugation $\left(\sigma_{i} \tau_{\infty} \sigma_{i}^{-1}\right)$ of $K(u)$ is independent of the choice of the embedding $\sigma_{i}$. More precisely, it acts by -1 on the unit $u$. It thus follows that $K(u)$ is a CM field. Therefore, the splitting field of $\widetilde{P}_{D}(x)$ lies inside the largest CM subfield of $K(f \infty)$.

In Gro81, Gross made the conjecture that certain $p$-units lying in $K(f \infty)$, the so-called Gross-Stark p-units, are related to the first derivative of a $p$-adic zeta function vanishing with order one at $s=0$. The author thinks that the units constructed in this paper are not new since they are expected to be written in terms of Gross-Stark p-units. For a partial result going in this direction see Theorem 5.2 of Cha07b.

The maximal CM subfield of $K(f \infty)$. Let $K=\mathbb{Q}(\sqrt{D})$. For any integer $f>0$, let $\mathcal{O}_{K}(f)^{\times}$(resp. $\left.\mathcal{O}_{K}(f \infty)^{\times}\right)$be the group of units (resp. totally positive units) of $\mathcal{O}_{K}$ which are congruent to 1 modulo $f$.

The next proposition determines the maximal $\mathrm{CM}$ subfield $L_{C M}^{D}$ of $K(f \infty)$ in the case where $n=1$. It turns out that in this case $\left[K(f \infty): L_{C M}^{D}\right]=2$. Let

$$
\operatorname{rec}_{K(f \infty) / K}: G_{K(f \infty) / K} \rightarrow I_{K}(f) / P_{K, 1}(f \infty)
$$

be the reciprocity map given by class field theory.

Proposition 7.1. Suppose that $\left[\mathcal{O}_{K}(f)^{\times}: \mathcal{O}_{K}(f \infty)^{\times}\right]=1$. Then the largest $C M$ field $L_{C M}^{D}$ which is contained in $K(f \infty)$ corresponds under $r e c_{K}(f \infty) / K$ to

$$
I_{K}(f) /\left\langle P_{K, 1}(f \infty),(f-1) \mathcal{O}_{K}\right\rangle .
$$

In other words, the prime ideals in $K$ which split completely in $L_{C M}^{D}$ are precisely the prime ideals in the group $\left\langle P_{K, 1}(f \infty),(f-1) \mathcal{O}_{K}\right\rangle$.

Proof. This is an easy application of class field theory. For the details see Section 17 of Cha. 
Symmetries of the $p$-adic valuations of the roots of $\widetilde{P}_{D}(x)$. Assume that $K(f \infty)$ is a totally complex CM field. Let $\mathfrak{c}=(1+f \sqrt{D})$ and let

$$
[\mathfrak{c}] \in I_{K}(f) / P_{K, 1}(f \infty) .
$$

Because $K(f \infty)$ is totally complex we have that $[\mathfrak{c}]$ is an ideal class of order 2 . Let $[\mathfrak{a}] \in I_{K}(f) / P_{K, 1}(f \infty)$ where $\mathfrak{a}$ is chosen to be an integral ideal. In Proposition 6.1 of [Cha07a], it is shown that

$$
3 \zeta^{*}(\delta, \mathfrak{a}, 0)=v_{p}(u([\mathfrak{a}])),
$$

where $\zeta^{*}(\delta, \mathfrak{a}, s)$ is a certain Archimedean zeta function attached to a binary quadratic form which represents the ideal class $[\mathfrak{a}]$. Let $\tau_{\mathfrak{c}}=r e c_{K(f \infty) / K}(\mathfrak{c})$ be the complex conjugation on $K(f \infty)$ corresponding to the ideal class $\mathfrak{c}$ and let $K(f \infty)^{\tau_{\mathfrak{c}}}$ be the subfield of $K(f \infty)$ fixed by $\tau_{\mathfrak{c}}$. One can show that

$$
\zeta^{*}(\delta, \mathfrak{a}, s)+\zeta^{*}(\delta, \mathfrak{c a}, s),
$$

can be written as a linear combination of $L$-functions of the form $L(s, \chi)$ where $\chi$ varies over various characters of the Galois group $\operatorname{Gal}\left(K(f \infty)^{\tau_{\mathrm{c}}} / K\right)$. Since at least one of the two real places of $K$ always split in $K(f \infty)^{\tau_{\mathrm{c}}}$ we may deduce from equation (3.1) of [Tat84] that $L(0, \chi)=0$. Therefore,

$$
\zeta^{*}(\delta, \mathfrak{a}, 0)=-\zeta^{*}(\delta, \mathfrak{c a}, 0)
$$

The previous identity is in harmony with the fact that the restriction of $\tau_{\mathfrak{c}}$ to the subfield $L_{C M}^{D} \subseteq K(f \infty)$ corresponds to the complex conjugation, and so one expects that

$$
v_{p}\left(u([\mathfrak{a}])^{\tau_{\mathfrak{c}}}\right)=v_{p}(u([\mathfrak{a c}]))=-v_{p}(u([\mathfrak{a}])) .
$$

The first equality is a consequence of Conjecture 2.2 applied to $u([\mathfrak{a}])$ and the second one comes from (7.4).

There are other symmetries that one can deduce from (7.3). Let $\sigma$ be the nontrivial automorphism of $K$. Then a direct computation shows that

$$
3 \zeta^{*}\left(\delta, \mathfrak{a}^{\sigma}, 0\right)=3 \zeta^{*}(\delta, \mathfrak{a}, 0) .
$$

From (7.5), one deduces that for every ideal class $[\mathfrak{a}] \in I_{K}(f) / P_{K, 1}(f \infty)$ such that $[\mathfrak{a}] \neq\left[\mathfrak{a}^{\sigma}\right]$, the valuation $v_{p}(u([\mathfrak{a}]))$ appears twice in the list of valuations of all the roots of $\widetilde{P}_{D}(x)$.

Let $\mathfrak{a}=r \Lambda_{\tau}$ where $r \in \mathbb{Z}_{>0},(r, f)=1$, and let $\tau \in K-\mathbb{Q}$ be chosen so that it is reduced with respect to $p$ and that $\tau-\tau^{\sigma}>0$. By definition of $u([\mathfrak{a}])$, we have

$$
v_{p}(u([\mathfrak{a}]))=\psi_{r}\left\{\infty \rightarrow \gamma_{\tau} \infty\right\} .
$$

As explained in Section 5.1, one has that $\psi_{r}\left\{\infty \rightarrow \gamma_{\tau} \infty\right\}=\psi_{(f-r)}\left\{\infty \rightarrow \gamma_{\tau} \infty\right\}$. It follows from this that if the two ideals $r \Lambda_{\tau},(f-r) \Lambda_{\tau}$ are nonequivalent modulo $P_{K, 1}(f \infty)$, then the valuation $v_{p}(u([\mathfrak{a}]))$ appears twice in the list of valuations of all the roots of $\widetilde{P}_{D}(x)$.

These two phenomenona are visible in the tables. 


\section{RESULTS}

In Tables 1-5, we summarize the computations performed using the algorithm described in Section 6 for $f=3, N_{0}=4$ and $\delta=2[1,1]-3[2,1]+1[4,1] \in D(4,3)$ for the prime numbers $p \in\{7,13,17\}$. In the last table, we consider the divisor $\delta^{\prime}=2[1,1]-1[2,1] \in D(4,3)$ for the prime number $p=7$.

The first column contains the values of admissible discriminants $D$ (in increasing order) with respect to the set of data $\left(p, f, N_{0}\right)$. The second column gives the structure of the abelian group $I_{K}(3) / P_{K, 1}(3 \infty)$ where $K=\mathbb{Q}(\sqrt{D})$. The third column gives the largest integer $n_{D} \mid 12$ as defined in (5.7). The fourth and fifth columns give the $p$-adic valuation of the various roots of the polynomials $P_{D}(x)$ and $\widetilde{P}_{D}(x)$ respectively. The sixth column gives the polynomial $\widetilde{P}_{D}(x)$ and, finally, the last column gives the degree over $K$ of the largest CM field $L_{C M}^{D}$ which is contained in $K(3 \infty)$.

Let $D$ be an admissible discriminant and let $K=\mathbb{Q}(\sqrt{D})$. For every entry of the tables, one can verify that the splitting field $M_{D}$ of $\widetilde{P}_{D}(x)$ generates a subfield of the maximum CM subfield $L_{C M}^{D}$ of $K(3 \infty)$. For many values of $D$, it turns out that $M_{D}=L_{C M}^{D}$. However, there are examples where $M_{D}$ is a proper subfield of $L_{C M}^{D}$. By varying the choice of the good divisor $\delta \in D(4,3)$, one can hope to find a polynomial $\widetilde{P}_{D}^{\delta}(x)$ which generates $L_{C M}^{D}$. For example, when $p=7$, $D=185(K=\mathbb{Q}(\sqrt{D}))$ and $\delta=2[1,1]-3[2,1]+1[4,1] \in D(4,3)$, the splitting field $M_{D}^{\delta}$ of the polynomial $\widetilde{P}_{D}^{\delta}(x)$ generates the field $K(\sqrt{-3})$ which has index 2 in $L_{C M}^{D}=K(3 \infty)$. However, if one takes the divisor $\delta^{\prime}=2[1,1]-1[2,1]$, then the splitting field $M_{D}^{\delta^{\prime}}$ of the polynomial $\widetilde{P}_{D}^{\delta^{\prime}}(x)$ generates the whole CM field $K(3 \infty)$.

For all the tables, the various roots of the polynomials were computed to a precision of $100 p$-adic digits. According to Magma, all the polynomials appearing in the tables are irreducible over $K$. Finally, the first and the last tables suggest that the divisor $\delta=2[1,1]-1[2,1]$ generates polynomials with smaller height than the ones associated to the divisor $\delta=2[1,1]-3[2,1]+1[4,1]$.

In the next two pages we include two examples where the polynomials have a very large degree, namely 40 and 44 respectively. In order to compute these polynomials we computed $p$-adic moments up to an accuracy of $200 p$-adic digits for $p=7$. The running time for part 1 of our algorithm was approximately 2 and a half days and the running time for part 2 of the algorithm was about 2 minutes for each of these two polynomials. We hope that these two examples will convince the reader of the efficiency of $p$-adic methods for the construction of large noncyclotomic class fields of real quadratic number fields.

The coefficients of the two polynomials below were computed to an accuracy of $200 p$-adic digits where $p=7$. The divisor that was used is $\delta^{\prime}=2[1,1]-1[2,1] \in$ $D(4,3)[1$

\footnotetext{
${ }^{1}$ There is an available version of this program on the author's website.
} 
Case $D=3601$ : For $D=3601$ we have $I_{K}(3) / P_{K, 1}(3 \infty) \simeq(\mathbb{Z} / 2 \mathbb{Z})^{2} \times \mathbb{Z} / 20 \mathbb{Z}$. The index $n_{D}=6$. The valuations of the roots of $P_{D}(x)$ are given by

$$
\begin{aligned}
& \pm 0, \pm 0, \pm 0, \pm 0, \pm 0, \pm 0, \pm 0, \pm 0, \pm 0, \pm 0, \pm 0, \pm 0, \pm 0, \pm 0, \pm 0, \pm 0, \pm 0, \pm 0, \pm 12, \pm 12 \\
& \pm 12, \pm 12, \pm 12, \pm 12, \pm 12, \pm 12, \pm 12, \pm 12, \pm 24, \pm 24, \pm 24, \pm 24, \pm 24, \pm 24, \pm 24, \pm 24 \\
& \pm 60, \pm 60, \pm 120, \pm 120 .
\end{aligned}
$$

The valuations of the roots of $\widetilde{P}_{D}(x)$ are given by

$$
\begin{aligned}
& \pm 0, \pm 0, \pm 0, \pm 0, \pm 0, \pm 0, \pm 0, \pm 0, \pm 0, \pm 0, \pm 0, \pm 0, \pm 0, \pm 0, \pm 0, \pm 0, \pm 0, \pm 0, \pm 2, \pm 2 \\
& \pm 2, \pm 2, \pm 2, \pm 2, \pm 2, \pm 2, \pm 2, \pm 2, \pm 4, \pm 4, \pm 4, \pm 4, \pm 4, \pm 4, \pm 4, \pm 4, \pm 10, \pm 10, \pm 20, \pm 20 .
\end{aligned}
$$

The polynomial $\widetilde{P}_{D}(x)=g(x)^{2}$ where $g(x)$ equals

$$
x^{40}+\frac{1}{2 \cdot 7^{20}}(5386147326578097 \sqrt{D}+207236645766181591) x^{39}
$$

$+\frac{1}{2 \cdot 7^{56}}(153558388573347724642946528771807029960865534085 \sqrt{D}+12723038523780880046611973581444130785857271298067) x^{23}$ $+\frac{1}{7^{56}}(50807431609947631125051566032925799024802663746 \sqrt{D}+2533044332851700130351918461623231284046855781910) x^{22}$ $+\frac{1}{2 \cdot 7^{56}}(113795138736219397785757493206104930488305304007 \sqrt{D}+4618457462806256994306551710691804199961882681281) x^{21}$

$+\frac{1^{56}}{2 \cdot 7^{56}}(144551247256263857906880429168658966997967332019 \sqrt{D}+13360629954966427818640981484624345128004585188989) x^{20}$ $+\frac{1}{2 \cdot 7^{56}}(113795138736219397785757493206104930488305304007 \sqrt{D}+4618457462806256994306551710691804199961882681281) x^{19}$ $+\frac{1}{7^{56}}(50807431609947631125051566032925799024802663746 \sqrt{D}+2533044332851700130351918461623231284046855781910) x^{18}$ $+\frac{1}{2 \cdot 7^{56}}(153558388573347724642946528771807029960865534085 \sqrt{D}+12723038523780880046611973581444130785857271298067) x^{17}$ $+\frac{1}{2 \cdot 7^{56}}(115939323749664354434256194415944494035381894369 \sqrt{D}+4236277040053129547273797735300018666039251471195) x^{16}$ $+\frac{1}{2 \cdot 7^{56}}(71015035950013129895850106646109479941337459013 \sqrt{D}+4046479023085337586519647860995208476164110120947) x^{15}$ $+\frac{1}{7^{56}}(62507698081151507452632873743887953441201734394 \sqrt{D}+5213050803284019092585095740653772889498434999078) x^{14}$ $+\frac{1}{2 \cdot 7^{56}}(107548299643853035727274076195816661515728771285 \sqrt{D}+3248218038077319538872644475662288620464185757283) x^{13}$ $+\frac{1}{2 \cdot 7^{56}}(46852494939040584435202280714148480524576696307 \sqrt{D}+3465767207251108289978546281389120151050016430501) x^{12}$ $+\frac{1}{7^{54}}(807660418082278121456233385415633353967302559 \sqrt{D}+25324870141361107249627450998393352582296792889) x^{10}$ $+\frac{1}{2.7^{52}}(9267248418209320144281979836845014701375429 \sqrt{D}+901789626561211582928142769370162529509827699) x^{9}$ $+\frac{1}{7^{50}}(261993782501477490756768502428277675940034 \sqrt{D}+18371230311900350611113854054556566185831755) x^{8}$ $+\frac{1}{2 \cdot 7^{48}}(8541482645479761890406405906034776363459 \sqrt{D}+209142979662888011669696550382296756211701) x^{7}$ $+\frac{1}{7^{46}}(7918378474398293584229361117840128214 \sqrt{D}+2398690655608290472326300079935700002534) x^{6}$ $+\frac{1}{7^{42}}(27731428750738337585288836695453612 \sqrt{D}+1798162506696112787204938617769492092) x^{5}$ $+\frac{1}{7^{38}}(8086393053842005337165246829357 \sqrt{D}+300538763694637961561479766808169) x^{4}$ $+\frac{1}{2 \cdot 7^{34}}(-1814560196023965442732984899 \sqrt{D}+28745633511018881023481540491) x^{3}$ $+\frac{1}{7^{30}}(404439232967551932686865 \sqrt{D}+24134999603103953100140691) x^{2}$ $+\frac{1}{2 \cdot 7^{20}}(5386147326578097 \sqrt{D}+207236645766181591) x+1$.

The maximal CM subfield $L_{C M}^{D}$ of $K(3 \infty)$ has degree 40 over $K=\mathbb{Q}(\sqrt{D})$. One can show that the splitting field of $g(x)$ over $K$ generates $L_{C M}^{D}$. 
Case $D=4009$ : For $D=4009$ we have $I_{K}(3) / P_{K, 1}(3 \infty) \simeq(\mathbb{Z} / 2 \mathbb{Z})^{2} \times \mathbb{Z} / 22 \mathbb{Z}$. The index $n_{D}=6$. The valuations of the roots of $P_{D}(x)$ are given by

$$
\begin{aligned}
& \pm 6, \pm 6, \pm 6, \pm 6, \pm 6, \pm 6, \pm 6, \pm 6, \pm 6, \pm 6, \pm 6, \pm 6, \pm 6, \pm 6, \pm 6, \pm 6, \pm 6, \pm 6, \pm 6, \pm 6 \\
& \pm 6, \pm 6, \pm 6, \pm 6, \pm 6, \pm 6, \pm 18, \pm 18, \pm 18, \pm 18, \pm 18, \pm 18, \pm 18, \pm 18, \pm 30, \pm 30 \\
& \pm 42, \pm 42, \pm 42, \pm 42, \pm 54, \pm 54, \pm 102, \pm 102 .
\end{aligned}
$$

The valuations of the roots of $\widetilde{P}_{D}(x)$ are given by

$$
\begin{aligned}
& \pm 1, \pm 1, \pm 1, \pm 1, \pm 1, \pm 1, \pm 1, \pm 1, \pm 1, \pm 1, \pm 1, \pm 1, \pm 1, \pm 1, \pm 1, \pm 1, \pm 1, \pm 1, \pm 1, \pm 1 \\
& \pm 1, \pm 1, \pm 1, \pm 1, \pm 1, \pm 1, \pm 3, \pm 3, \pm 3, \pm 3, \pm 3, \pm 3, \pm 3, \pm 3, \pm 5, \pm 5 \\
& \pm 7, \pm 7, \pm 7, \pm 7, \pm 9, \pm 9, \pm 17, \pm 17 .
\end{aligned}
$$

The polynomial $\widetilde{P}_{D}(x)=h(x)^{2}$ where $h(x)$ equals

$$
\begin{gathered}
x^{44}+\frac{1}{2 \cdot 7^{11}}(-18083580929505 \sqrt{D}-1683479771586043) x^{43} \\
+\frac{1}{7^{20}}(882986280097086347715 \sqrt{D}+83043737465881297344683) x^{42}
\end{gathered}
$$

$+\frac{1}{27^{33}}(-1455503002801812490524001143 \sqrt{D}-149138916026369082536146959421) x^{4}$

$+\frac{1}{740}(-505154134472175097845310077745242 \sqrt{D}+1615444476609430006435400947167257) x^{40}$

$+\frac{1}{2 \cdot 7^{45}}(78681284133763816074357597697948664653 \sqrt{D}+3576944753269386377644372085206031082887) x^{39}$

$+\frac{1}{7^{48}}(-19060017849622566903121116827884077061987 \sqrt{D}-908404190368000289048692988742689414918157) x^{38}$

$+\frac{1}{27^{51}}(6527201848726351225462448563106549153895509 \sqrt{D}+192265402900742856962693884669242369336056351) x^{37}$

$+\frac{1}{7^{5}(1347300756430898654301644877922557116003166403 \sqrt{D}+130139703486600653637967785624331106536920547772) x^{36}}$

$+\frac{1}{7^{57}}(-1065414369855526912122886499352008081773452501549 \sqrt{D}-82278056580321294170048638908677655276722046156427) x^{35}$

$+\frac{1}{7^{3}}(5250657992079346827935811137551905111290970857160 \sqrt{D}+445583380315630066491436661796908056664712626434050) x^{34}$

$\frac{1}{7^{58}}(26390123288632441347688347727467857012812066536115 \sqrt{D}+189773801184803595917081720662536460162227086066081) x^{33}$
$+\frac{1}{2 \cdot 7^{35}}(263952$

$+\frac{1}{2 \cdot 7^{60}}(-785809615492388726539322421771409427061464134401769 \sqrt{D}-39355563383612755514413412620378674545315357605420161) x^{32}$

$+\frac{1}{7^{0}}(2847844501928187913050036495156811982296177535635517 \sqrt{D}+146542207124131132944408485809910239771233663433885575) x^{31}$

$+\frac{1}{7^{62}}(-10092353871264794059738471534598322043507601878888853 \sqrt{D}-422786540596136931766077345257191457275107502151907295) x^{30}$

$+\frac{1}{7^{63}}(5924954828356328581966705862122876296305948013621360 \sqrt{D}-952104293660630260231699440896044971296736802553596048) x^{29}$

$+\frac{1}{2 \cdot 7^{6 \mathrm{6s}}}(370425958563019393963049071743787029713224574275518265 \sqrt{D}+38682293409411590531254702339187212935119709344171097551) x^{28}$

$+\frac{1}{7^{65}}(-3061350319607726901254732247821146712936322574079924686 \sqrt{D}-230884480601744779011471786488965566016482904136753347698) x^{27}$

$+\frac{1}{7^{56}}(38919681795479813051998258415941132901062216791783538171 \sqrt{D}+2656420921084302970784204143815164660041106966415452418181) x^{26}$

$+\frac{1}{7^{67}}(-290994712197834526589415640145596357075265996002096270397 \sqrt{D}-18853031240794636355232259973145024740720042628687043995951) x^{25}$

$+\frac{1}{7^{68}}(405691821755111731772381739247785537620076759165868015250 \sqrt{D}+25964311663114103810539853252939446987514882096419971840780) x^{24}$

$+\frac{1}{2 \cdot 7^{65}}(29960582977620375345845139763278757179777830926555596910549 \sqrt{D}+1930976418077915981129667363992751302846384334062211900501327) x^{23}$ $+\frac{1}{2 \cdot 7^{70}}(-331699140706350088038643449873088482869148687891633226451785 \sqrt{D}-21454773446473761621685822154741055461062820129485462770228411) x^{22}$

$+\frac{1}{2 \cdot 7^{65}}(29960582977620375345845139763278757179777830926555596910549 \sqrt{D}+1930976418077915981129667363992751302846384334062211900501327) x^{21}$

$+\frac{1}{T^{\mathbb{S}}}(405691821755111731772381739247785537620076759165868015250 \sqrt{D}+25964311663114103810539853252939446987514882096419971840780) x^{20}$

$+\frac{1}{7^{66}}(38919681795479813051998258415941132901062216791783538171 \sqrt{D}+2656420921084302970784204143815164660041106966415452418181) x^{18}$

$+\frac{1}{7^{65}}(-3061350319607726901254732247821146712936322574079924686 \sqrt{D}-230884480601744779011471786488965566016482904136753347698) x^{17}$

$+\frac{1}{2 \cdot 7^{6 \mathrm{tx}}}(370425958563019393963049071743787029713224574275518265 \sqrt{D}+38682293409411590531254702339187212935119709344171097551) x^{16}$

$+\frac{1}{7^{63}}(5924954828356328581966705862122876296305948013621360 \sqrt{D}-952104293660630260231699440896044971296736802553596048) x^{15}$

$+\frac{1}{7^{2}}(-10092353871264794059738471534598322043507601878888853 \sqrt{D}-422786540596136931766077345257191457275107502151907295) x^{14}$

$+\frac{1}{7^{61}}(2847844501928187913050036495156811982296177535635517 \sqrt{D}+146542207124131132944408485809910239771233663433885575) x^{13}$

$+\frac{1}{2.7^{\text {to }}}(-785809615492388726539322421771409427061464134401769 \sqrt{D}-39355563383612755514413412620378674545315357605420161) x^{12}$

$+\frac{1}{2 \cdot 7^{5 y}}(26390123288632441347688347727467857012812066536115 \sqrt{D}+189773801184803595917081720662536460162227086066081) x^{11}$

$+\frac{1}{7^{53}}(5250657992079346827935811137551905111290970857160 \sqrt{D}+445583380315630066491436661796908056664712626434050) x^{10}$

$+\frac{1}{7^{2}}(-1065414369855526912122886499352008081773452501549 \sqrt{D}-82278056580321294170048638908677655276722046156427) x^{9}$

$+\frac{1}{7^{57}}(1347300756430898654301644877922557116003166403 \sqrt{D}+130139703486600653637967785624331106536920547772) x^{8}$

$+\frac{1}{27^{51}}(6527201848726351225462448563106549153895509 \sqrt{D}+192265402900742856962693884669242369336056351) x^{7}$

$+\frac{1}{7^{48}}(-19060017849622566903121116827884077061987 \sqrt{D}-908404190368000289048692988742689414918157) x^{6}$

$+\frac{1}{2.745}(78681284133763816074357597697948664653 \sqrt{D}+3576944753269386377644372085206031082887) x$

$+\frac{1}{7^{4 \pi}}(-505154134472175097845310077745242 \sqrt{D}+1615444476609430006435400947167257) x^{4}$

$+\frac{1}{2 \cdot 7^{3}}(-1455503002801812490524001143 \sqrt{D}-149138916026369082536146959421) x^{3}$

$+\frac{1}{7^{20}}(882986280097086347715 \sqrt{D}+83043737465881297344683) x^{2}$

$+\frac{1}{2 \cdot 7^{17}}(-18083580929505 \sqrt{D}-1683479771586043) x+1$

The maximal CM subfield $L_{C M}^{D}$ of $K(3 \infty)$ has degree 44 over $K=\mathbb{Q}(\sqrt{D})$. One can show that the splitting field of $h(x)$ over $K$ generates $L_{C M}^{D}$. 


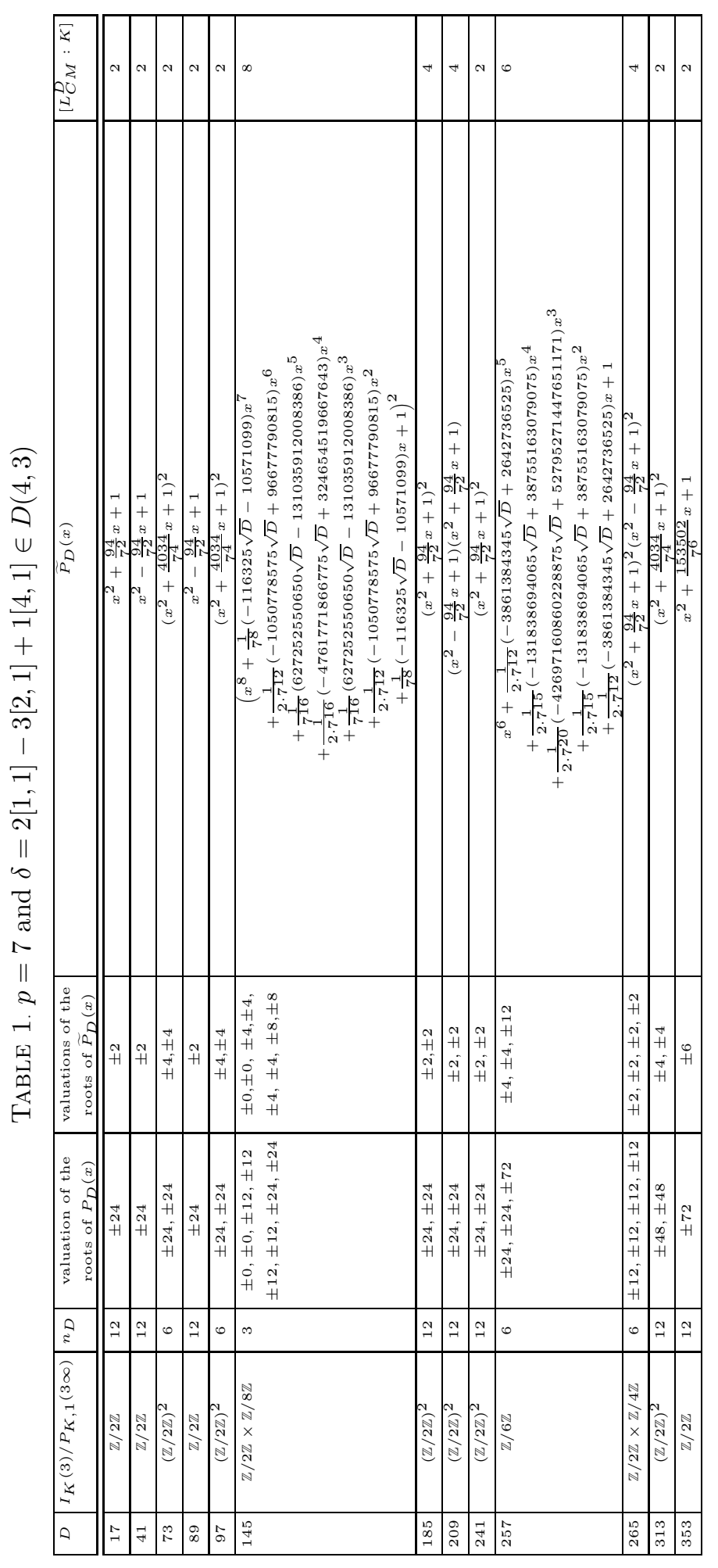




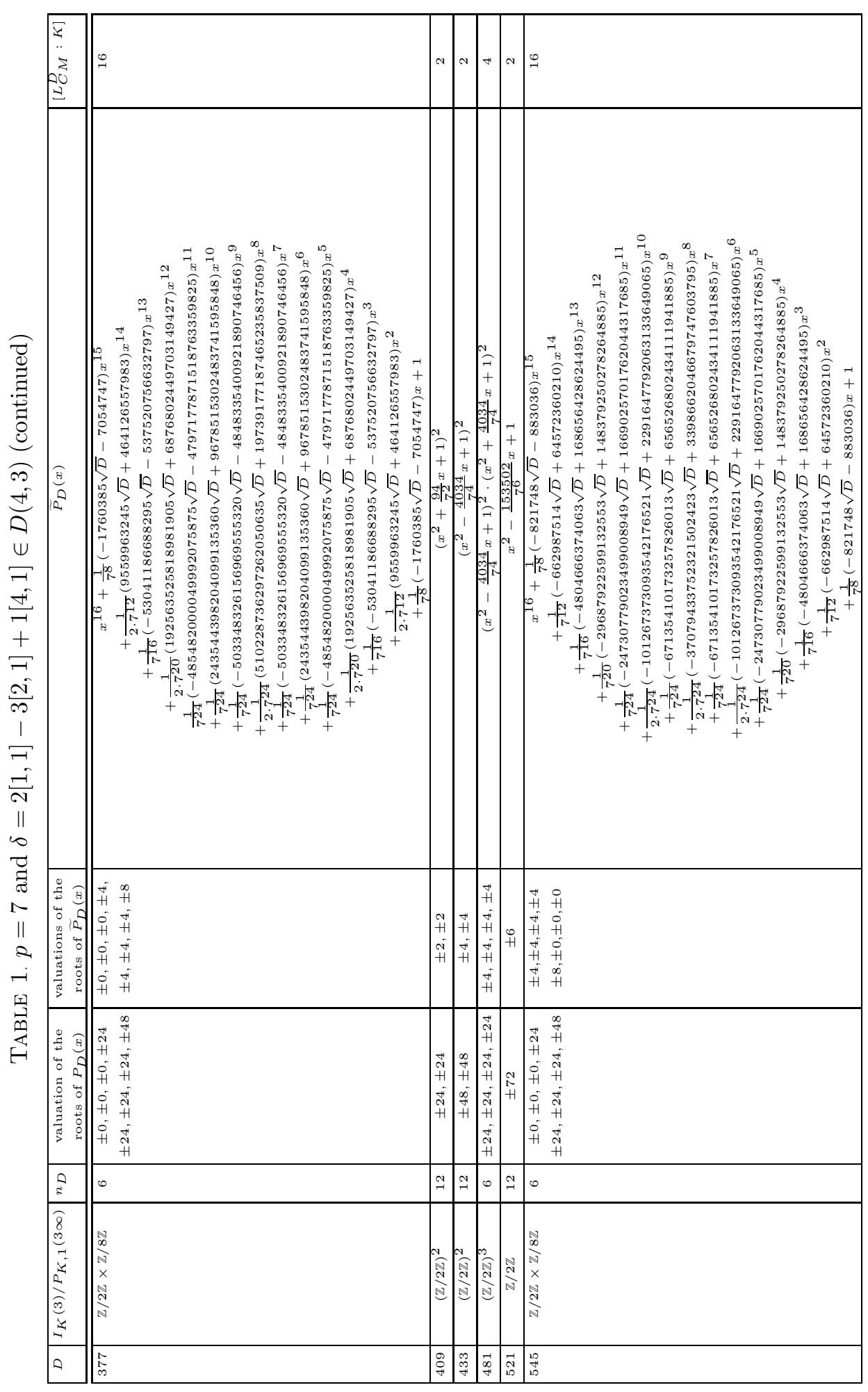




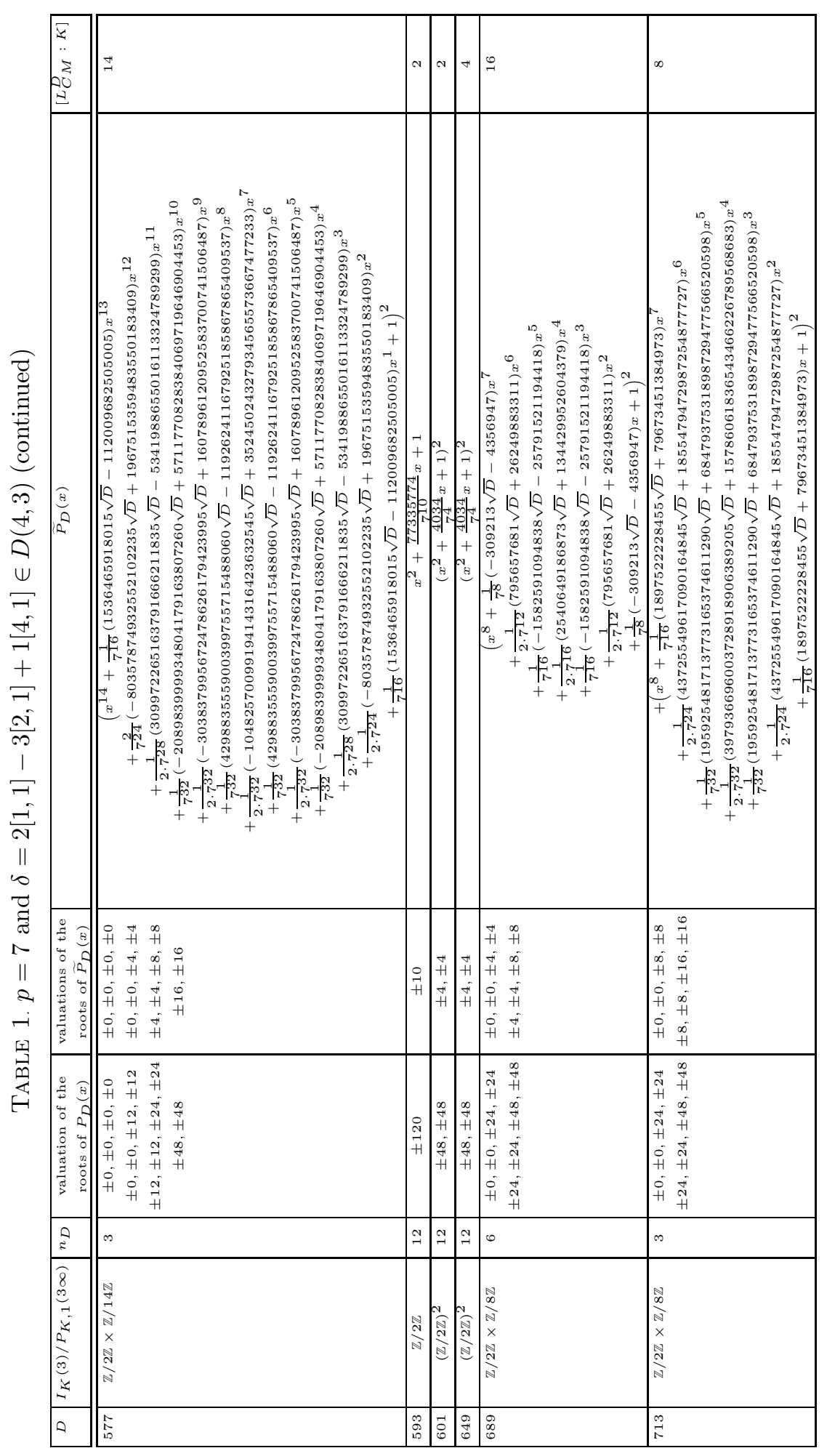




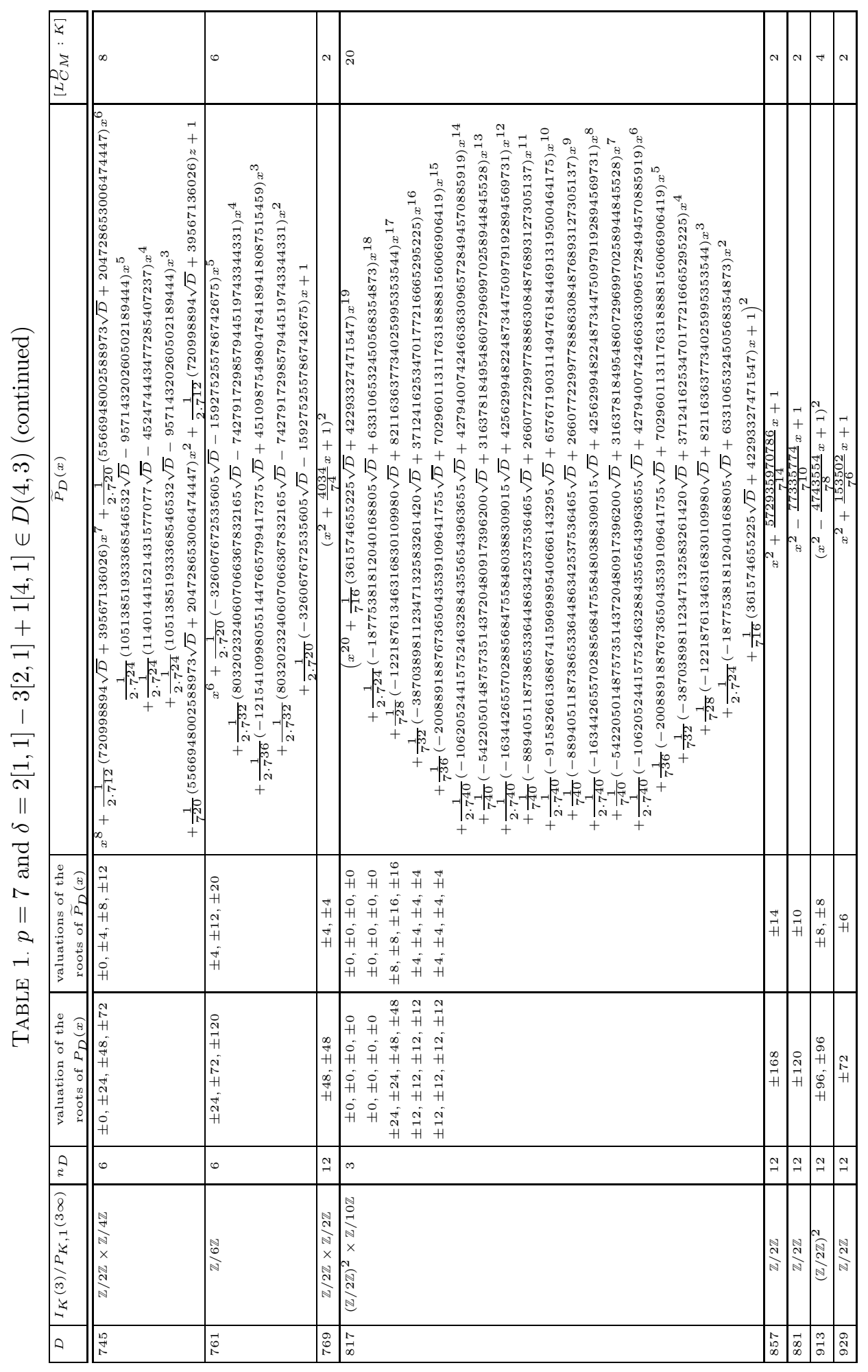




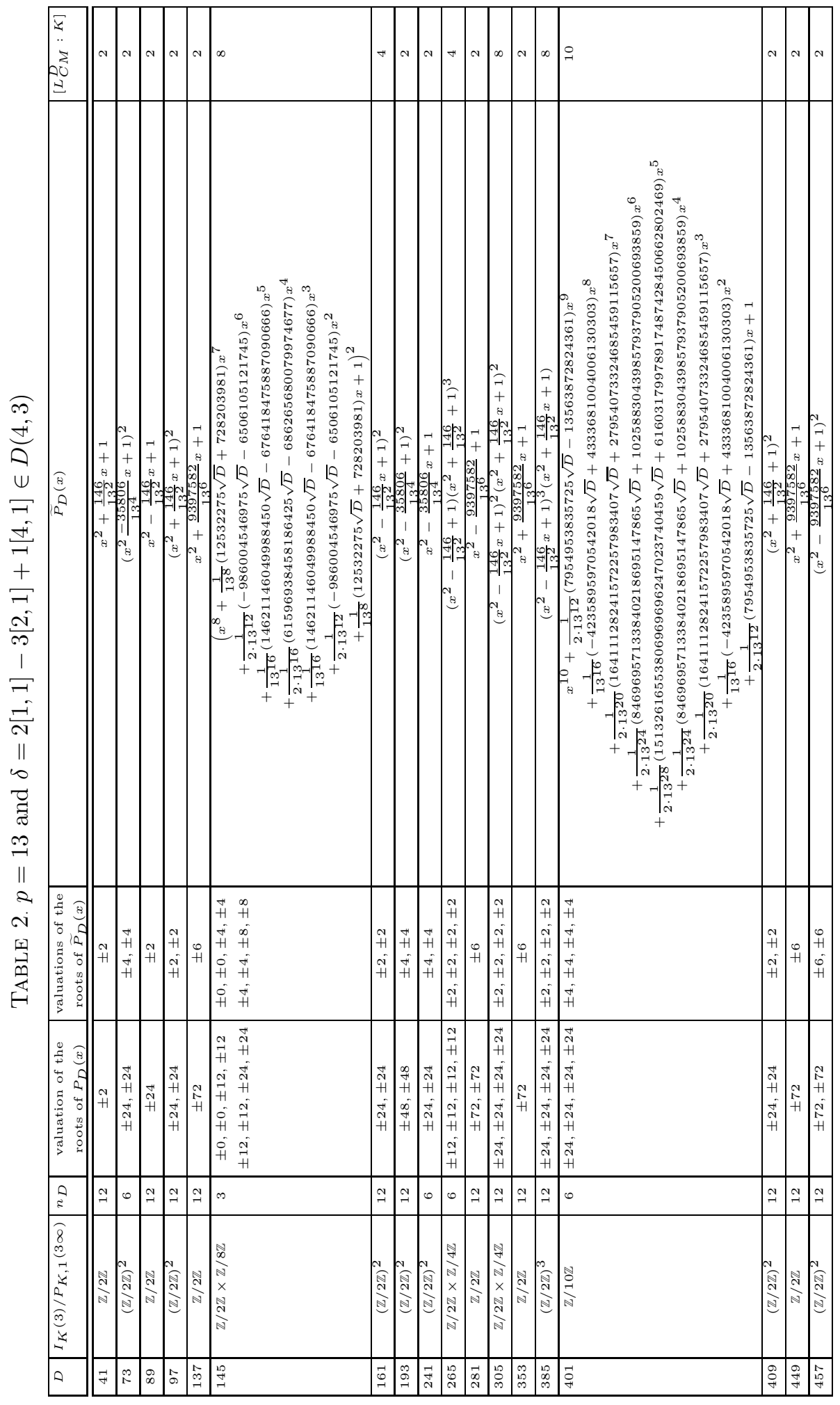




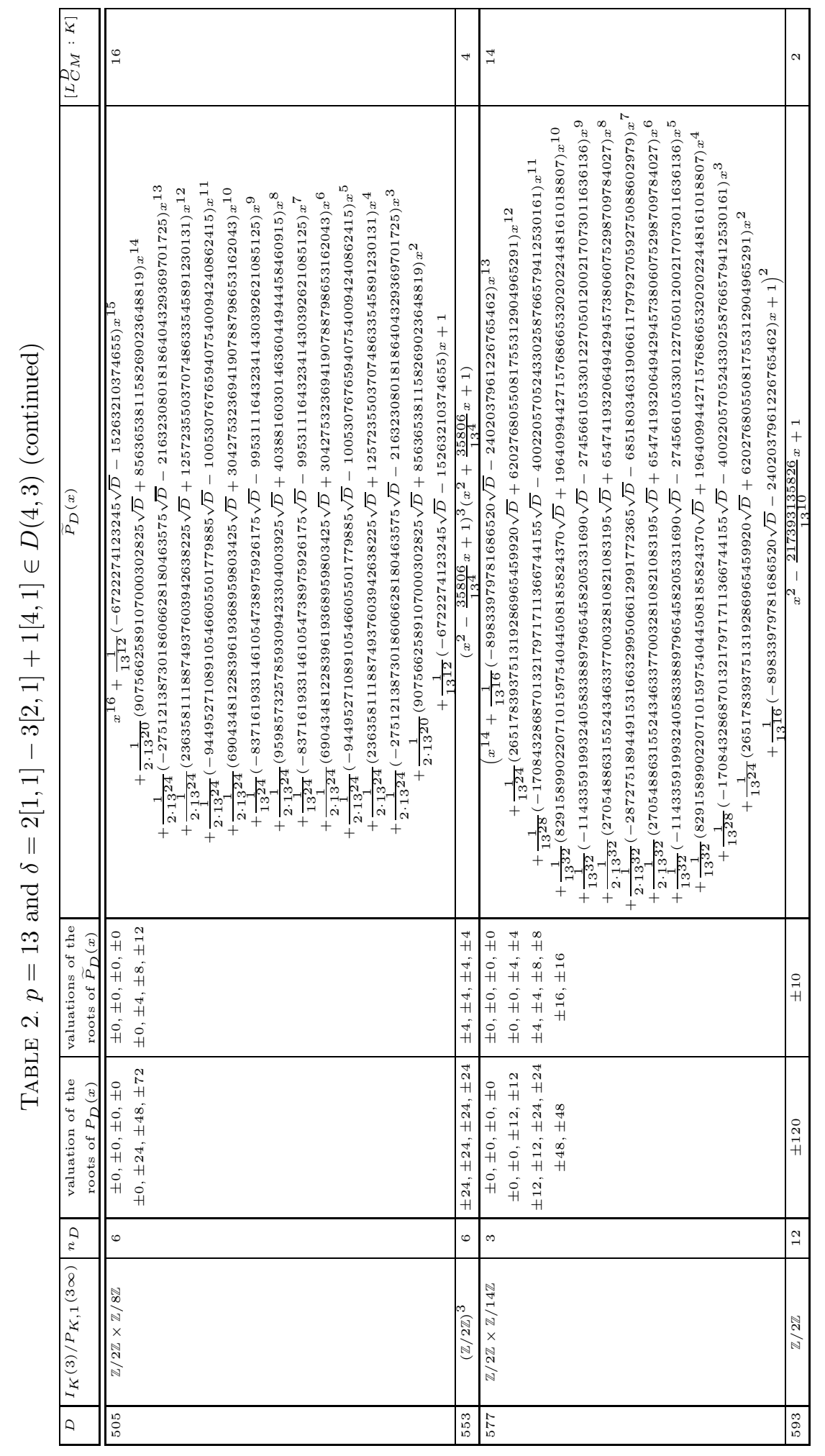




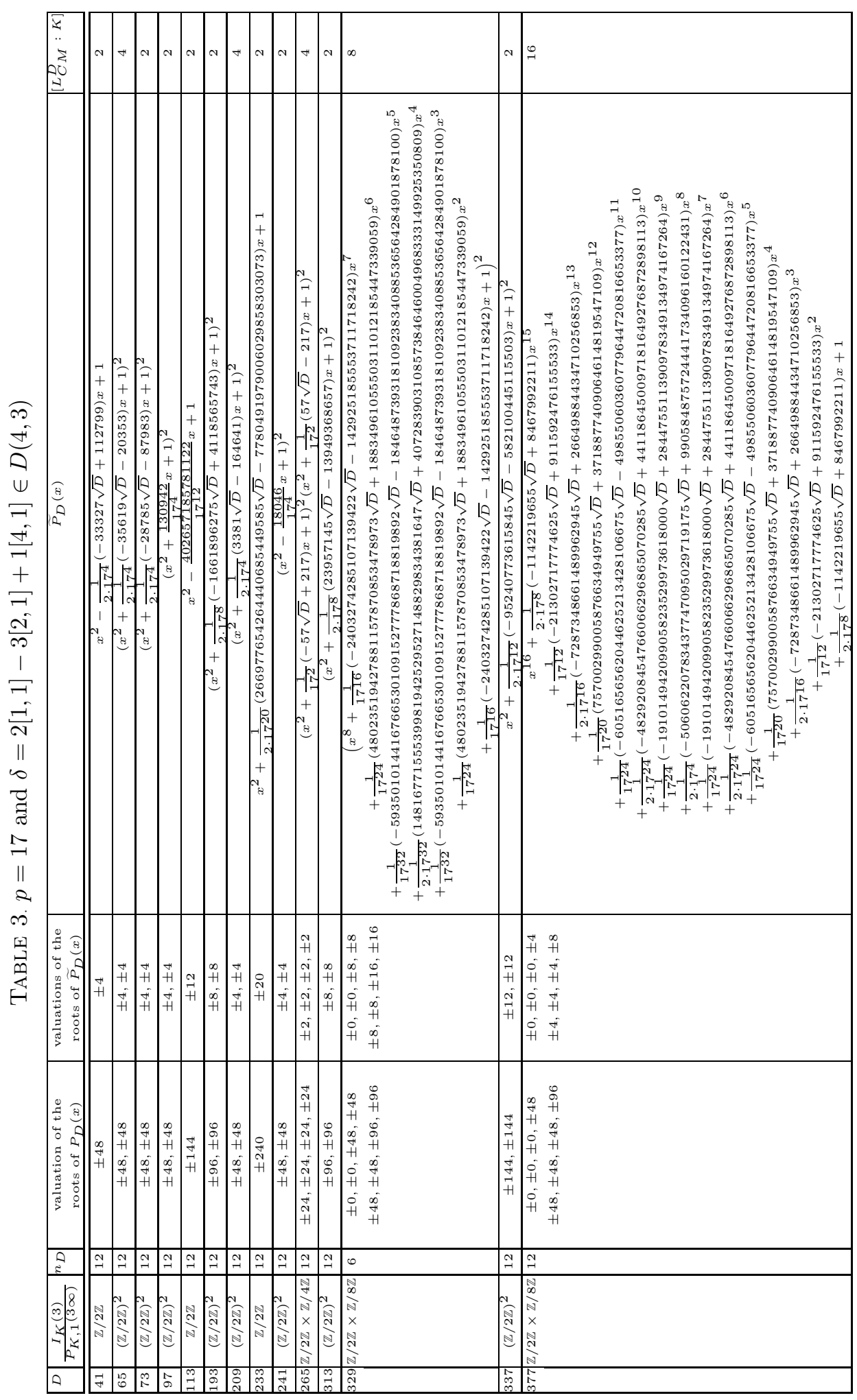



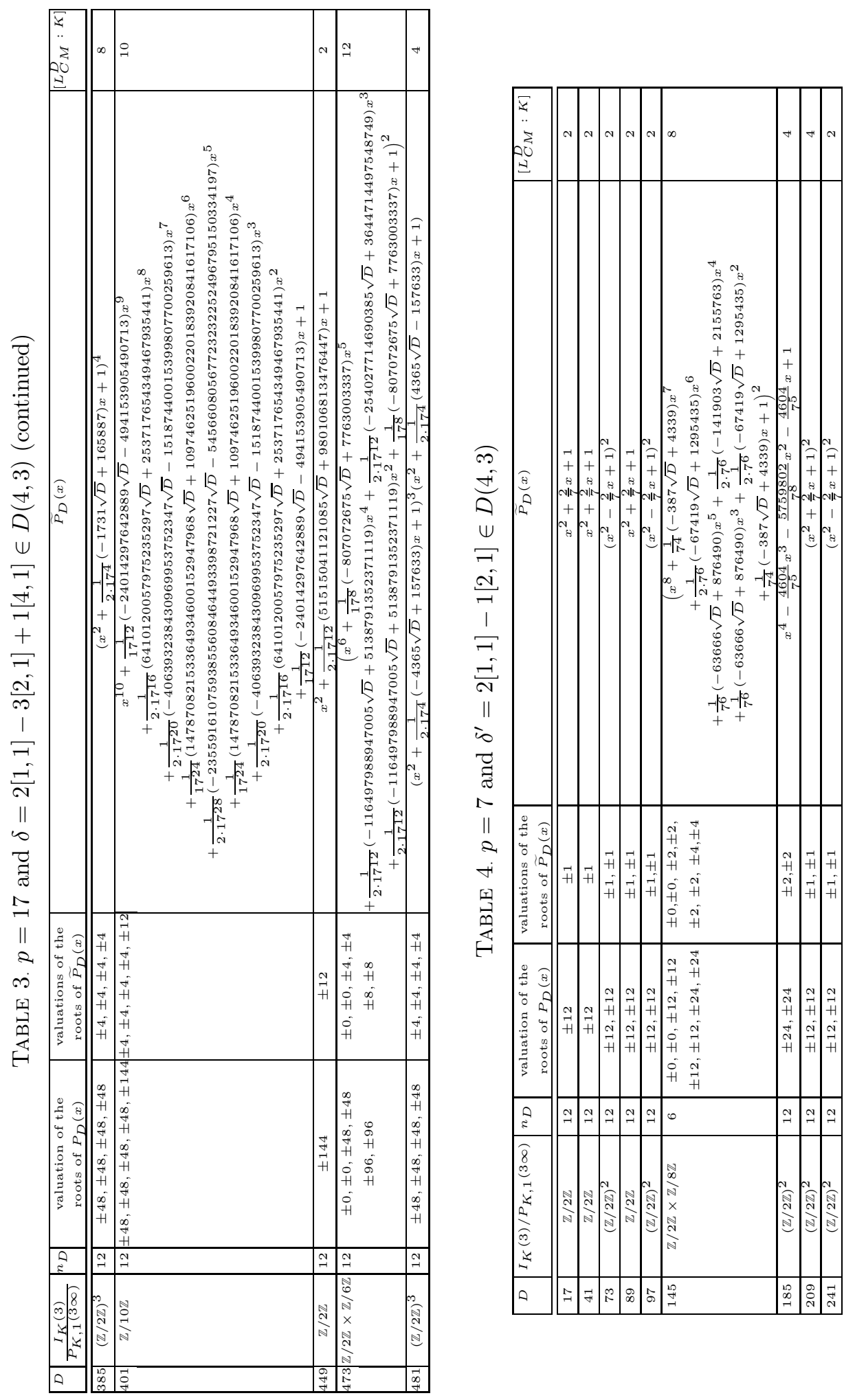
HUGO CHAPDELAINE

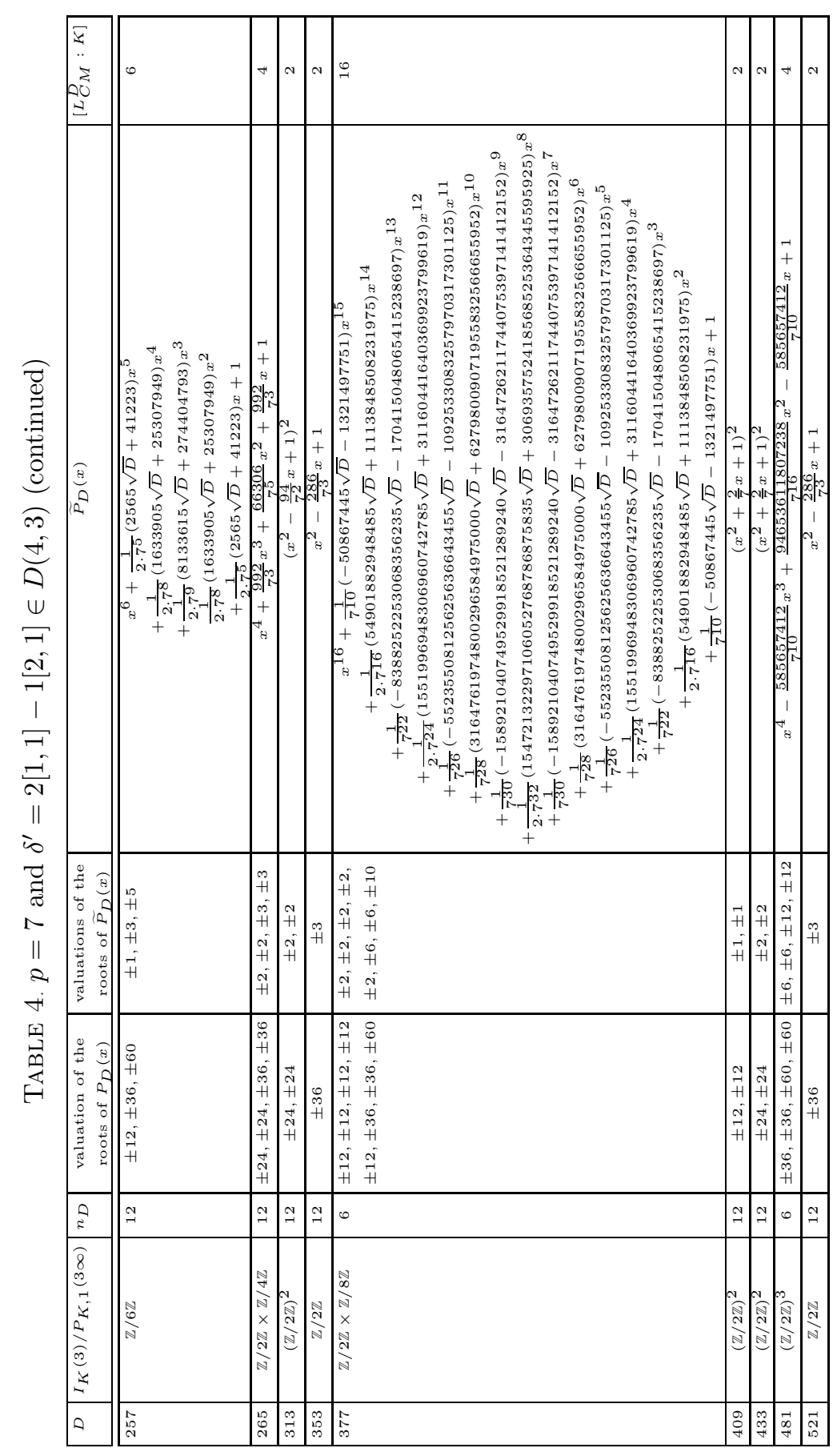




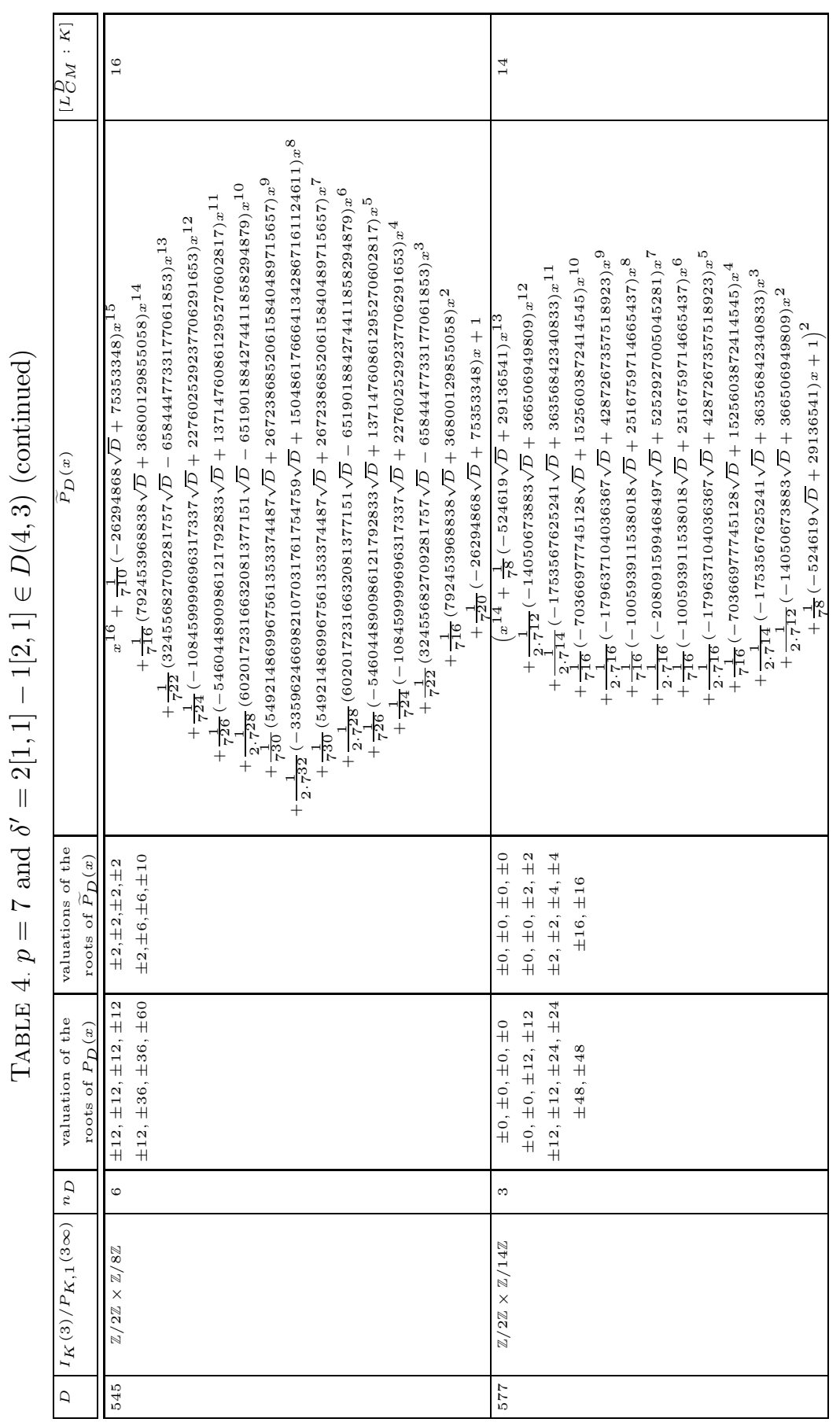




\begin{tabular}{|c|c|c|c|c|c|c|}
\hline 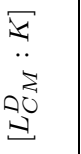 & $\| \sim$ & $\sim$ & + & $\stackrel{\bullet}{-1}$ & $\infty$ & $\infty$ \\
\hline$\frac{\beta}{8}$ & 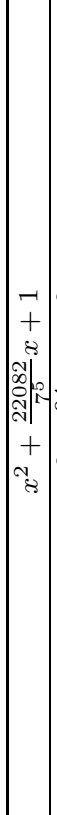 & 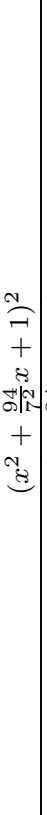 & 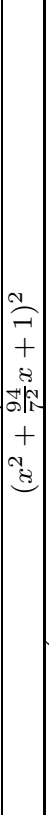 & 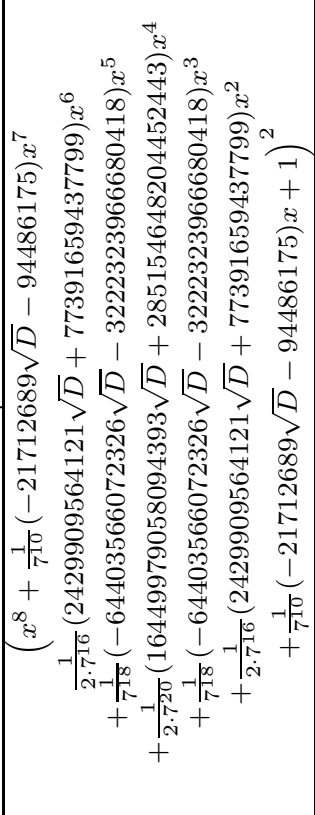 & 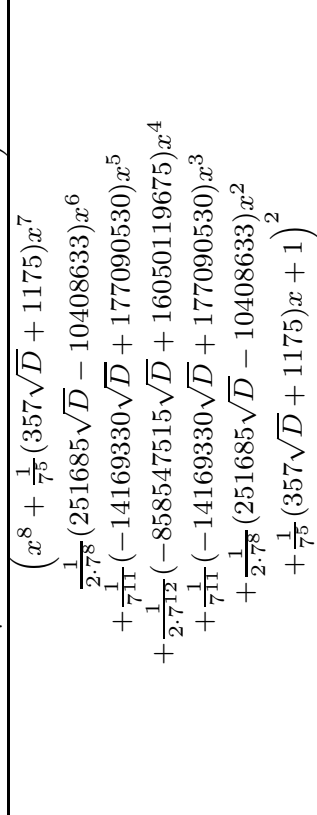 & 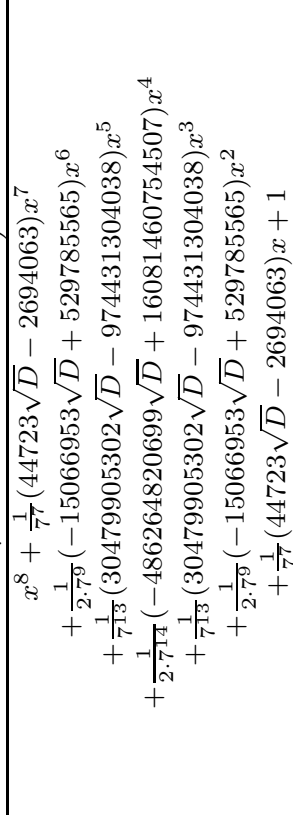 \\
\hline 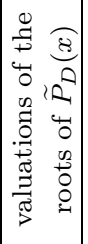 & $\mid \begin{array}{l}20 \\
+1\end{array}$ & 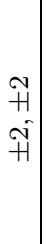 & $\mid \begin{array}{l}\text { I } \\
\text { in } \\
\text { in }\end{array}$ & 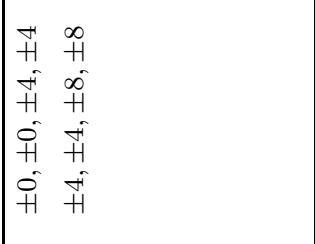 & 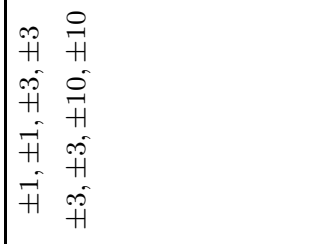 & 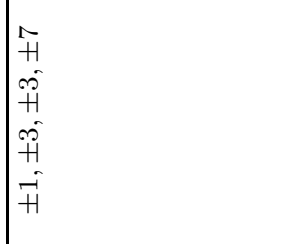 \\
\hline 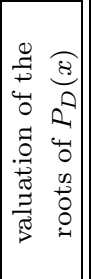 & $\mid \begin{array}{c}0 \\
0 \\
+1\end{array}$ & $\begin{array}{l}\vec{N} \\
+ \\
\vec{H} \\
\vec{H}\end{array}$ & $\begin{array}{l}\vec{N} \\
H \\
H \\
\vec{N} \\
H\end{array}$ & 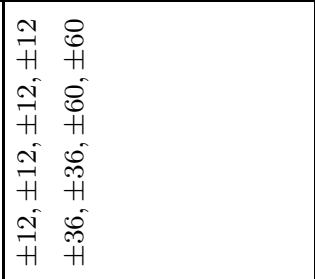 & 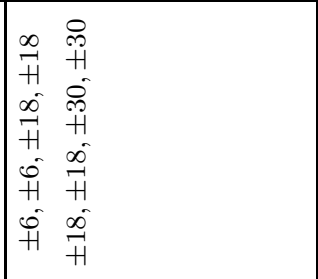 & 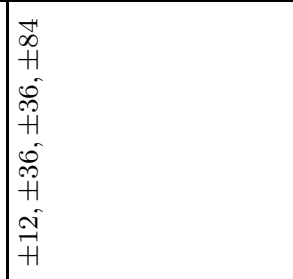 \\
\hline$\stackrel{\vec{\theta}}{E}$ & $\approx$ & 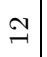 & $\approx$ & 0 & 0 & 0 \\
\hline 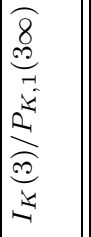 & $\frac{\mathbb{N}}{\mathbb{N}}$ & $\begin{array}{l}\mathbb{N} \\
\mathbb{N} \\
\mathbb{N}\end{array}$ & 零 & $\mid \begin{array}{l}\mathbb{N} \\
\infty \\
\mathbb{N} \\
x \\
\mathbb{N} \\
N \\
\mathbb{N}\end{array}$ & $\begin{array}{l}\mathbb{N} \\
\mathbb{N} \\
\times \\
\mathbb{N} \\
\mathbb{N} \\
\mathbb{N}\end{array}$ & $\begin{array}{l}\mathbb{N} \\
\mathbb{N} \\
\times \\
\times \\
\mathbb{N} \\
\mathbb{N} \\
\mathbb{N}\end{array}$ \\
\hline$\theta$ & 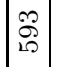 & 8 & | & 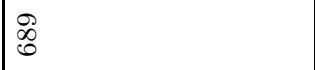 & $\ddot{m}$ & 㝵 \\
\hline
\end{tabular}


COMPUTATION OF $p$-UNITS IN RAY CLASS FIELDS

\begin{tabular}{|c|c|c|c|}
\hline 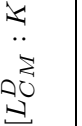 & $\| 0$ & $\sim$ & ลิ \\
\hline & 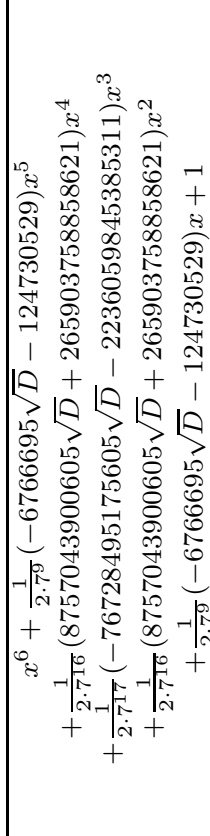 & 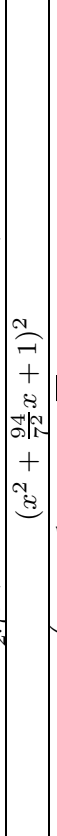 & 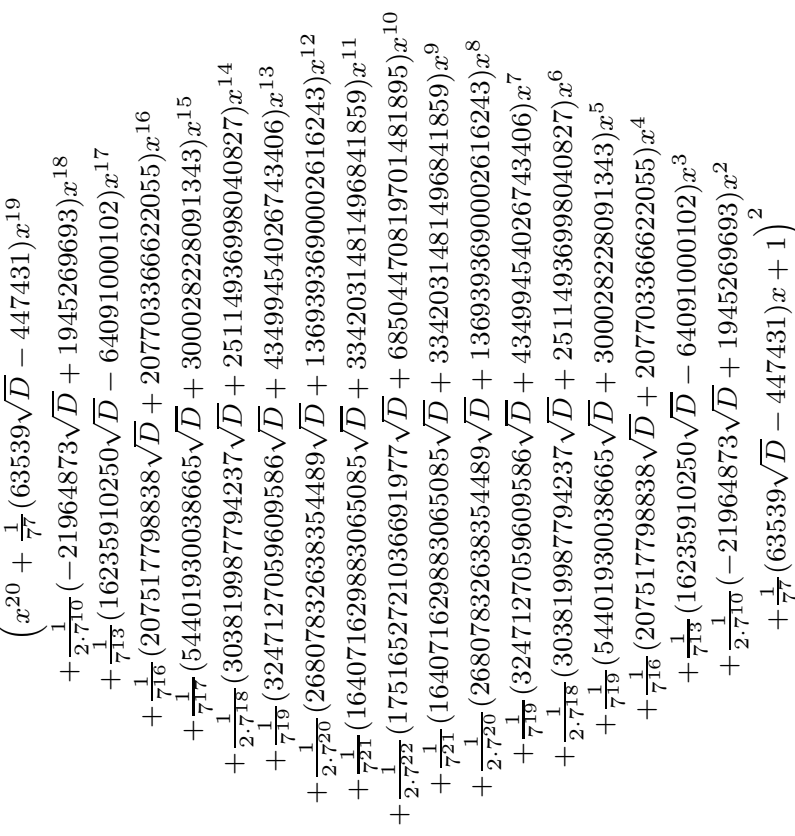 \\
\hline 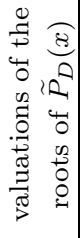 & 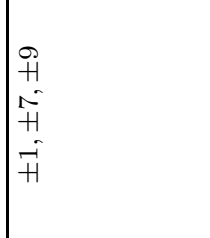 & 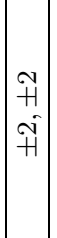 & 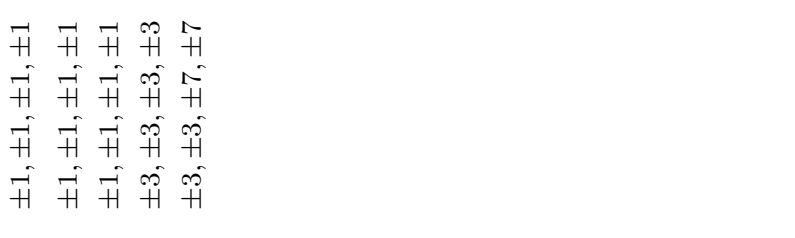 \\
\hline 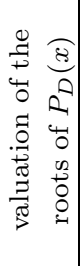 & 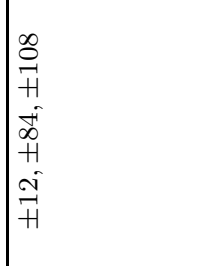 & 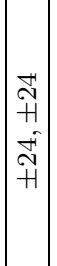 & 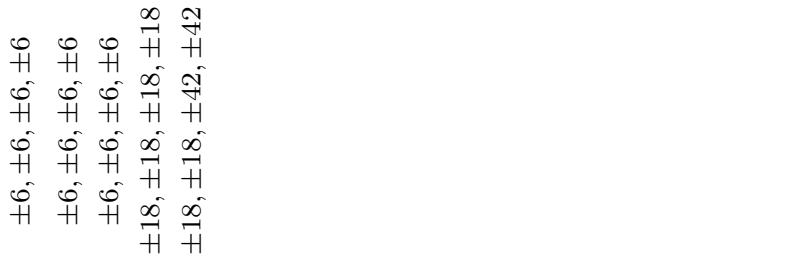 \\
\hline$\stackrel{\ominus}{ }$ & $\|_{0}$ & 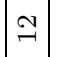 & 0 \\
\hline 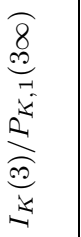 & $\| \frac{\mathbb{N}}{\mathbb{N}}$ & $\mid \begin{array}{c}\mathbb{N} \\
\mathbb{N} \\
\times \\
\mathbb{N} \\
\mathbb{N} \\
\mathbb{N}\end{array}$ & 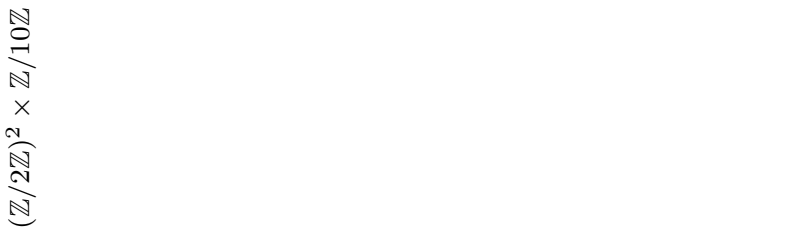 \\
\hline$\theta$ & $\overrightarrow{0}$ & $\mid \stackrel{0}{\circ}$ & $\stackrel{-}{\infty}$ \\
\hline
\end{tabular}




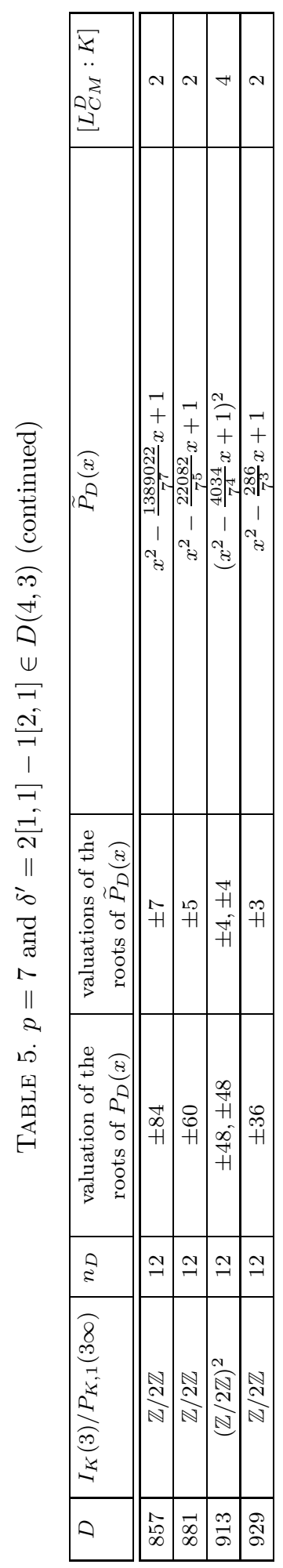




\section{REFERENCES}

[Cha] H. Chapdelaine. Elliptic units in ray class fields of real quadratic number fields, version with a few corrections and supplements. available at http://www.mat. ulaval.ca/fileadmin/Pages_personnelles_des_profs/hchapd/thesis_final.pdf.

[Cha07a] H. Chapdelaine. $p$-units in ray class fields of real quadratic number fields. accepted for publication in Compositio, 1:1-34, 2007.

[Cha07b] H. Chapdelaine. Zeta functions twisted by additive characters, $p$-units and Gauss sums. International J. Number Theory, 1:1-40, 2007.

[Dar04] H. Darmon. Rational points on modular elliptic curves. AMS Publication, 2004. MR.2020572 (2004k:11103)

[Das07a] S. Dasgupta. Computations of elliptic units for real quadratic number fields. Canadian Journal of Mathematics, pages 553-574, 2007. MR2319158 (2008d:11054)

[Das07b] S. Dasgupta. Shintani zeta functions and Gross-Stark units for totally real fields. Duke Mathematical Journal (2), 143:225-279, 2008. MR2420508

[DD06] H. Darmon and S. Dasgupta. Elliptic units for real quadratic fields. Annals of Mathematics (2), 163:301-346, 2006. MR2195136 (2007a:11079)

[Gro81] B. Gross. p-adic L-series at $s=0$. J. Fac. Sci. Univ. Tokyo, 28:979-994, 1981. MR656068 (84b:12022)

[Gro88] B. Gross. On the values of abelian $L$-functions at $s=0$. J. Fac. Sci. Univ. Tokyo, 35:177-197, 1988. MR931448 (89h:11071)

[Tat84] J. Tate. Les conjectures de Stark sur les fonction L d'Artin en $s=0$. Birkäuser, Boston, 1984. MR782485 (86e:11112)

Département de Mathématiques et de Statistique, Université Laval, Québec, Canada G1K 7P4

E-mail address: hugo.chapdelaine@mat.ulaval.ca 